\title{
Seismic probe of transport processes in red giants
}

\author{
MarieJo Goupil* \\ LESIA, Observatoire de Paris, 92195 Meudon, France and \\ CNRS, PSL Research, Pierre et Marie Curie and Denis Diderot universities
}

\begin{abstract}
Seismic data obtained with the space photometric CoRoT and Kepler instruments have led to a unprecendently precise characterization- in terms of masses and ages- of a large sample of post main sequence stars (low mass subgiant and red giants). The high quality of the collected seismic data and the subsequent theoretical work for interpreting them brought up a series of issues which revealed that our knowledge of the internal properties of red giant stars remains quite limited. Two such important issues are discussed here, namely mixing beyond the convective core of helium burning red giant stars and evolution of internal angular momentum for post main sequence stars. This includes how they were diagnosed and what are the resulting improvements in our understanding regarding these issues (or rather how far we are from a proper understanding and realistic modelling of the structure and evolution of post main sequence stars).
\end{abstract}

Keywords: stars: oscillations - stars: interiors - stars: evolution - asteroseismology - stars: red giants

\section{Introduction}

Space-based observations by CoRoT (Baglin et al., 2006, 2016) and Kepler (Borucki, 2010, 2018) revealed that low mass subgiants and red giants (understanding here with masses below $1.4-1.5 M_{\odot}$ ) oscillate with nonradial modes which, similarly to the Sun, are excited by the convective motions of the envelope. Unlike the Sun and due to their evolved structure, they actually show a rich spectrum of mixed modes (e.g., Dziembowski, 1971; Scuflaire, 1974; Aizenman et al., 1977). These modes have significant amplitudes both in the core - mostly localized at and below the hydrogen-burning shellwhere the dominant restoring force is buoyancy (g-cavity) as well as in the outer envelope where the dominant restoring force is the pressure gradient (p-cavity) (Dziembowski et al., 2001; Dupret et al., 2009). This property enables us to study the inner properties of the star quasi directly. However the richness of the mixed mode frequency spectrum comes with a high complexity which makes disentangling the various modes and measurements of their frequencies highly challenging. Fortunately, the excited range of modes lies in a regime where the modes closely follow an asymptotic behavior in the g-cavity. This is less true in the p-cavity but deviations from asymptotics remain acceptable for first order investigations. The result of such seismic analyses was tremensdouly wealthy: -determination of seismic masses and ages with unprecedent precision; -the unvaluable segregation between giant stars ascending the giant branch, stars in the primary and secundary clumps which was not possible previoulsy with classical observations only - measurement of the core rotation profile and its time evolution. These advances led to a total renewal of the galactic archeology field. They also brought up a serious problem with the modelling of internal angular momentum transport. This wealthy crop

*mariejo.goupil@obspm.fr 


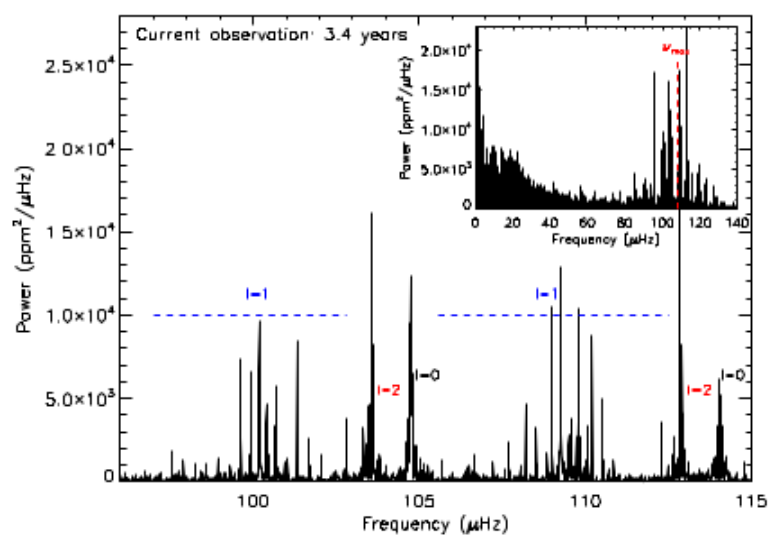

Figure 1: Power spectrum of the red giant KIC 10977979 from a 3.4 year-long lightcurve. The frequency peaks associated with $\ell=$ 0, 1,2 modes can be clearly seen as indicated in the figure (credit Benomar et al., 2013).

of information was already reviewed (Chaplin \& Miglio, 2008; Mosser \& Miglio, 2016; Hekker \& Christensen-Dalsgard, 2017). Here I will concentrate on two unsolved issues in the modelling of low mass subgiant and red giant stars: mixing beyond the convective core (CC) of red clump (RC) stars (Sect.3) and internal angular momentum (AM) in post main sequence stars (PoMS) (Sect.4). The first problem was already known but was confirmed by the seismology of these stars while the second one was an unexpected outcome of the PoMS seismic analyses. Before addressing these issues, I must introduce the tools that led to these discoveries. Hence I start in Sect.2, by briefly reviewing the main seismic observables and diagnotics accessible to PoMS stars and the theoretical basis that allows the interpretation of these observations. Some conclusions are drawn in Sect.5.

\section{Seismic constraints for evolved low mass stars}

As already mentioned, the power spectrum of a low mass PoMS star exhibits a complex frequency pattern. A typical exemple of an observed pattern of a set of $\ell=0,1,2$ modes is shown in Fig.1. For sake of notation, lets recall a few pre-requisite general statements. One interprets such a frequency or power spectrum in a linear adiabatic normal mode framework (Cox, 1980; Unno et al. 1989; Gough, 1993). The observed frequencies are associated with normal modes of the stellar resonant cavity. The low mass PoMS stars are mainly slow rotators; one then usually assumes that the equilibrium state keeps its spherical symmetry. Accordingly the geometry of each linear (eigen-)mode can be described by means of a single spherical harmonics and the associated frequency $\nu$ and pulsation $\omega=2 \pi \nu$ are usually labelled with the degree $\ell$ and the azimuthal order $m$ of the spherical harmonics. In the following only $\ell=1$ modes will be considered. The $\ell, m$ indices will be omitted unless necessary. The frequency must also be labelled with a radial order $n$ associated with the number of nodes of the eigenfunction in the radial direction. When necessary, one also usually distinguishes the number of nodes in the g-cavity and in the p-cavity and denotes them as $n_{g}$ and $n_{p}$ respectively. For the observed modes of evolved stars, $k_{r} \Delta r_{g}>>1$ in the g-cavity where $k_{r}$ is the radial wavenumber of the mode and $\Delta r_{g}$ the extension of the inner g-cavity then $n_{g}>>1$. In the p-cavity, $k_{r} \Delta r_{p} \geq 1$ with $\Delta r_{p}$ the extension of the outer p-cavity and typically $n_{p} \sim 6-10$.

A first information comes from the large frequency separation defined for axisymetric dipolar modes as:

$$
\Delta \nu_{n}=\nu_{n}-\nu_{n-1}
$$

$\Delta \nu_{n}$ increases with the frequency $\nu_{n}$ and reaches at high frequency a nearly constant value $\Delta \nu$ which 


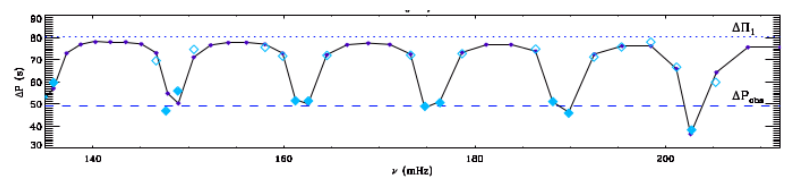

Figure 2: Period separations, $\Delta P_{n}$, as a function of the frequency $\nu$ for the red giant KIC 9882316. The modes with the largest period separations $\Delta P_{n}$ are $g$ dominated modes and the modes with the smallest $\Delta P_{n}$ are p-dominated modes. Each cyan diamond corresponds to an observed mode of the star. The g-mode spacing $\Delta \Pi_{1}$ is indicated by the dotted horizontal line (credit Mosser et al. 2012).

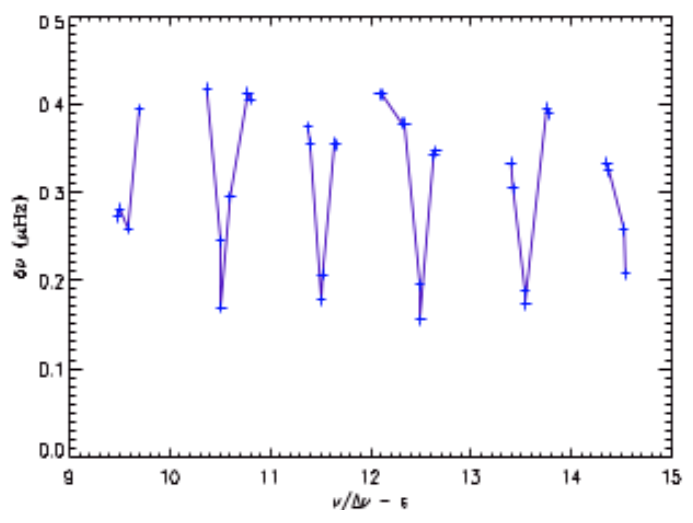

Figure 3: Rotational splittings as a function of the frequency normalized to the mean large separation for the Kepler star KIC 5356201 (data from Beck et al. 2012) (credit Goupil et al. 2013).

is closely related to the asymptotic (theoretical) large frequency separation defined as

$$
\Delta \nu_{a}=\left(2 \int_{0}^{R} \frac{d r}{c_{s}}\right)^{-1}
$$

where $c_{s}$ is the sound speed and $R$ the stellar radius.

A second seismic diagnostic comes from the observed period separation, $P_{n}$ defined as:

$$
\Delta P_{n}=P_{n}-P_{n-1}=\frac{1}{\nu_{n}}-\frac{1}{\nu_{n-1}}
$$

where $P_{n}$ is the oscillation period of a dipole mode with radial order $n$. The period separation can be related to the asymptotic (theoretical) period spacing which is defined as

$$
\Delta \Pi_{1}=\frac{2 \pi^{2}}{\sqrt{2}}\left(\int_{r_{a}}^{r_{b}} \frac{N}{r} d r\right)^{-1}
$$

The period separation is then known to be strongly related to the evolution stage and age of the red giants (Bedding et al. 2011; Mosser et al. 2011, 2012a, 2014).

The third main observable is the rotational splitting:

$$
\delta \nu_{n}=\nu_{n, 1}-\nu_{n,-1}
$$

where the second indice refers to the azimutal number of the associated $\ell=1$ spherical harmonics. The measurement of rotational splittings enabled determining the mean core rotation of subgiant and red giant stars (Beck et al. 2012; Mosser et al 2012b; Deheuvels et al, 2012, 2014, 2015; Di Mauro 2016, 2018).

Examples for these last two observables are displayed in Fig. 2 and Fig. 3.

Before presenting the fourth observable, one must introduce mode inertia, $I(\nu)$, which is defined as:

$$
I(\nu)=\int_{0}^{R}\left(\xi_{r}^{2}+\Lambda \xi_{h}^{2}\right) 4 \pi \rho r^{2} d r
$$




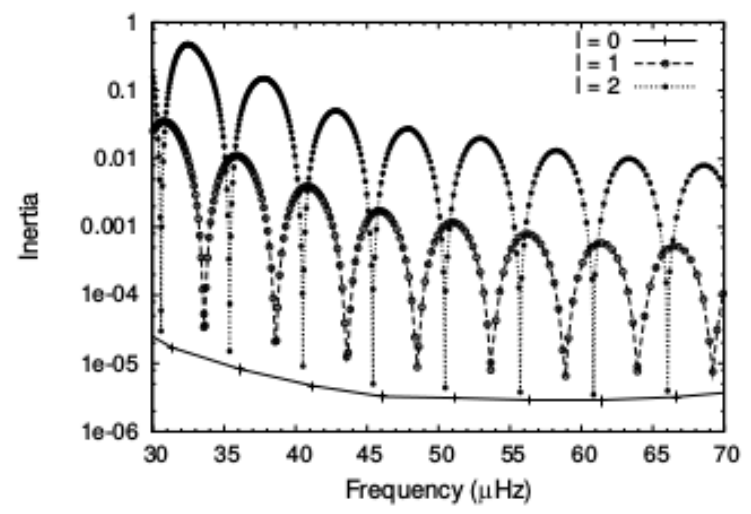

Figure 4: Mode inertia for a red giant model as a function of the mode frequency for $l=0,1,2$. Highest inertia are g-dominated modes. Lowest inertia are p-dominated modes. Intermediate inertia correspond to intermediate cases. The lowest curve connecting the dots represent the inertia of radial modes (credit Dupret et al., 2009).
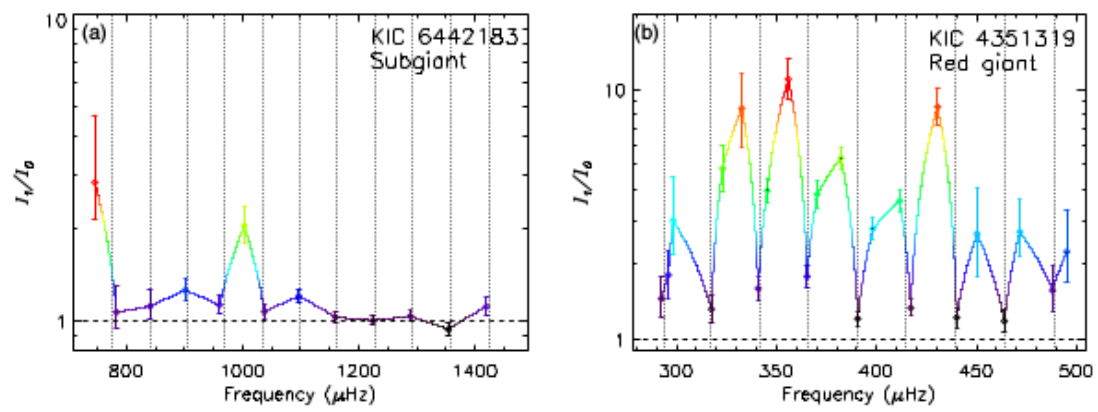

Figure 5: Observed core-to-total inertia ratio for the red giants (a) KIC 6442183 and (b) KIC 4351319 against frequency. The black, blue, red, and brown diamonds are symbols for $\ell=0,1,2,3$ respectively. Vertical dashed lines indicate the position of the nearly pure p-modes (from Benomar et al. 2014).

where $\Lambda=\ell(\ell+1)$ and $\xi_{r}$ and $\xi_{h}$ represent the vertical and horizontal displacement eigenfunctions respectively (Dziembowski et al., 2001). Fig. 4 shows that mode inertia significantly changes for mixed modes lying between two successives radial modes. Mode inertia, $I$, is much larger for gdominated modes than p-dominated modes due to the inward increase of the mass distribution.

The fourth observable discussed hereafter then is the inertia ratio (Goupil et al. 2013; Deheuvels et al. 2015; Benomar et al., 2014) defined as:

$$
\zeta(\nu)=\frac{I_{\text {core }}}{I}
$$

The observed variations of $\zeta$ with frequency are displayed in Fig.5 for two red giants. A good approximation is $I \approx I_{\text {core }}+I_{\text {env }}$ where one identifies the core with the g-mode cavity and the envelope with the p-mode cavity (neglecting inertia in the intermediate evanescent region). Accordingly, $\zeta \approx 1$ for g-dominated modes and $\zeta<<1$ for $\mathrm{p}$-dominated modes.

Interpretation of the observed period spacings and rotational splittings are easier when one realizes that these observables are both linearly related to mode inertia. More precisely, they follow the same behavior as a function of frequency than the inertia ratio $\zeta$ (Eq.7). This is discussed in more detailed in the next section. 

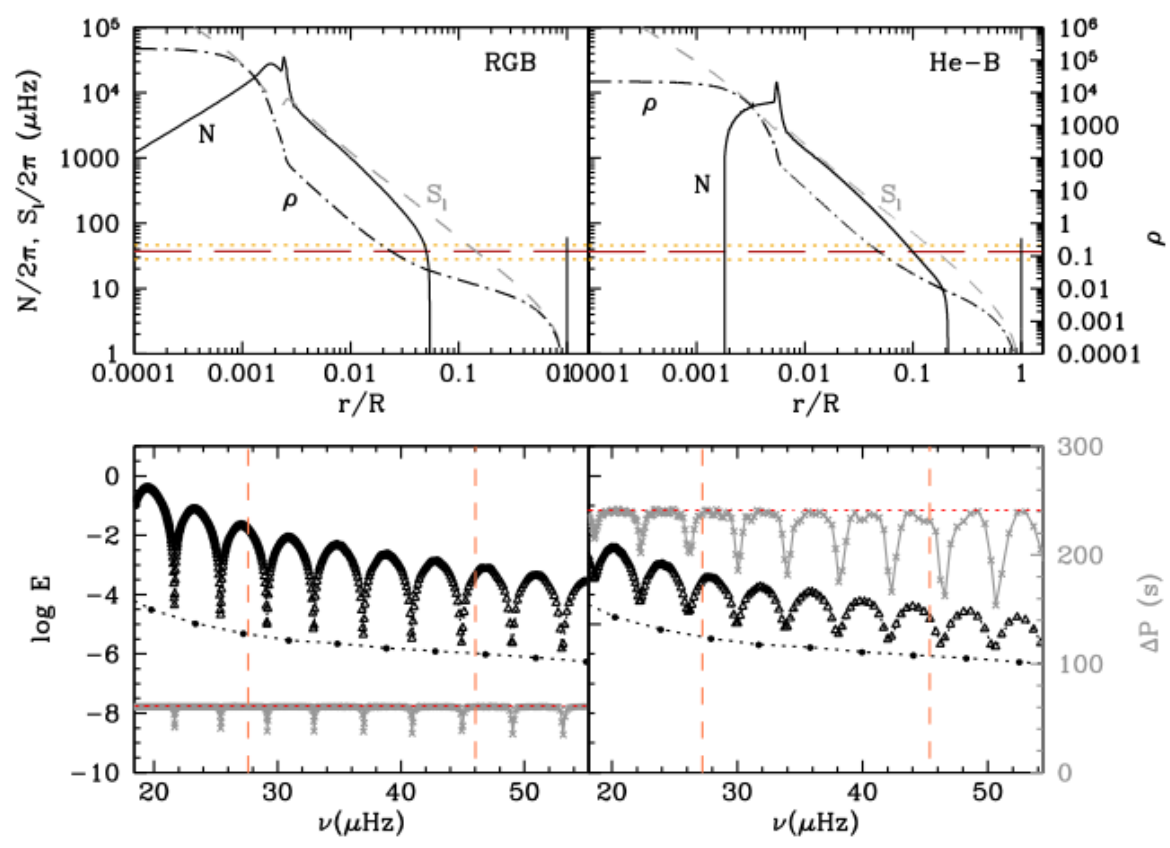

Figure 6: Top: Characteristics frequencies $N, S_{1}$ and density distribution for dipole modes for a $1.5 M_{\odot}, 12 R_{\odot}$ stellar model for a red giant (left) and a red clump star (right). The typical frequency range of excited modes for each star is shown by the horizontal dotted lines. The three turning points $\left(k_{r}=0\right)$ for these models are located at the intersection of this range and either $N$ or $S_{1}$. Bottom: inertia as a function of the frequency for $\ell=0$ (circles) and 1 (triangles) modes. The vertical dashed lines represent the typical frequency range of excited modes for such stars. Highest inertia are g-dominated modes. Lowest inertia are p-dominated modes. Grey crosses connected with a dotted line represent the period separation $\Delta P_{n}=P_{n+1}-P_{n}$. The horizontal red thin-dashed line indicates the asymptotic period spacing $\Delta \Pi_{1}$ (credit Montalban et al., 2013).

\subsection{Asymptotics of mixed dipole modes for post main sequence stars}

The properties of the observed oscillations of subgiants and red giants can be well investigated with an asymptotic analysis of mixed modes. In that framework, the structure of the equilibrium model is represented by two characteristic frequencies, the Lamb, $S_{\ell}$, and the Brünt-Väissälä, $N$, frequencies defined respectively as

$$
S_{\ell}^{2}=\frac{\Lambda c_{s}^{2}}{r^{2}} \quad ; \quad N^{2}=g\left(\frac{1}{\Gamma_{1}} \frac{d \ln p}{d r}-\frac{d \ln \rho}{d r}\right)
$$

where $c_{s}$ is the sound speed; $\Lambda=\ell(\ell+1), \Gamma_{1}, g, p, \rho$ have their usual meanings (see for instance Montalban et al., 2013 and Montalban \& Noels, 2014). In the following, the inner g-cavity is located between the radii $r_{a}$ and $r_{b}\left(r_{a} \sim 0<<r_{b}\right)$. The outer p-cavity is delimited by the radii $r_{c}$ and $r_{d}$ $\left(r_{b}<r_{c}<<r_{d} \sim R\right)$. These resonant cavities are separated by an intermediate evanescent region (delimited by the radii $r_{b}, r_{c}$ ). The situation is well depicted in Fig.6 for a red giant and a subgiant star.

The resonant condition for those modes was first derived by Shibahashi (1979) (see also Unno et al (1989)) in the Cowling approximation and in the limit of a thick evanescent region

$$
\cot \theta_{g} \tan \theta_{p}=q
$$

where

$$
\theta_{p}=\int_{r_{c}}^{r_{d}} k_{r}(r, \omega) d r \quad ; \quad \theta_{g}=\int_{r_{a}}^{r_{b}} k_{r}(r, \omega) d r
$$


and $q$ is the coupling factor between the two trapping regions.

When contributions from regions close to the turning points of the resonant cavities are neglected, the radial wave number $k_{r}(r, \omega)$ is given by

$$
k_{r}^{2}(r, \omega) \approx \frac{1}{\omega^{2} c_{s}^{2}}\left(\omega^{2}-S_{\ell}^{2}\right)\left(\omega^{2}-N^{2}\right)
$$

The turning points $\left(k_{r}=0\right) r_{a}, r_{b}, r_{c}, r_{d}$ are then given by $\omega=S_{1}\left(r_{c}\right)=S_{1}\left(r_{d}\right)=N\left(r_{a}\right)=N\left(r_{b}\right)$.

G-cavity: Away from the turning points, $\omega<<S_{l}, N$. The phase $\theta_{g}$ for the g-cavity can be approximated as:

$$
\theta_{g} \approx \sqrt{\Lambda} \int_{r_{a}}^{r_{b}}\left(\frac{N^{2}}{\omega^{2}}-1\right)^{1 / 2} \frac{d r}{r} \approx \frac{\pi}{\nu \Delta \Pi_{1}}+\pi \epsilon_{g}\left(r_{a}, r_{b}, \omega\right)
$$

where $\nu=2 \pi / \omega$.

For a pure g-mode trapped in a g-cavity delimited by $r_{a}, r_{b}$, the resonant condition imposes:

$$
\int_{r_{a}}^{r_{b}} k_{r}(r, \omega) d r=\frac{\pi}{\nu \Delta \Pi_{1}}+\pi \epsilon_{g}\left(r_{a}, r_{b}, \omega\right)=n_{g} \pi
$$

For a g-dominated mixed mode, the influence of the p-cavity rather leads to define a frequency $\nu_{g}$ such that:

$$
\int_{r_{a}}^{r_{b}} k_{r}\left(r, \omega_{g}\right) d r=\frac{\pi}{\nu_{g} \Delta \Pi_{1}}+\pi \epsilon_{g}\left(r_{a}, r_{b}, \omega_{g}\right) \approx\left(n_{g}+\frac{1}{2}\right) \pi
$$

For later purposes, the phase $\theta_{g}$, Eq.12, is then conveniently rewritten as

$$
\theta_{g} \approx \frac{\pi}{\Delta \Pi_{1}}\left(\frac{1}{\nu}-\frac{1}{\nu_{g}}\right)+\left(n_{g}+\frac{1}{2}\right) \pi
$$

P-cavity: Away from the turning points, $\omega>>S_{l}, N$ and the phase in the p-cavity is written as :

$$
\theta_{p} \approx 2 \pi \nu \int_{r_{c}}^{r_{d}}\left(1-\frac{S_{1}^{2}}{\omega^{2}}\right)^{1 / 2} \frac{d r}{c_{s}}+\pi \epsilon_{p}\left(r_{c}, r_{d}, \omega_{p}\right)
$$

We now define $\nu_{p}$ the frequency of the mode as if it was not perturbed by the g-part i.e. given by the acoustic resonant condition

$$
2 \pi \nu_{p} \int_{r_{c}}^{r_{d}}\left(1-\frac{S_{1}^{2}}{\omega^{2}}\right)^{1 / 2} \frac{d r}{c_{s}}+\pi \epsilon_{p}\left(r_{c}, r_{d}, \omega_{p}\right) \approx n_{p} \pi
$$

For later purposes, the phase $\theta_{p}$, Eq. 15 is then conveniently rewritten as:

$$
\theta_{p} \approx 2 \pi\left(\nu-\nu_{p}\right) \int_{r_{c}}^{r_{d}}\left(1-\frac{S_{1}^{2}}{\omega^{2}}\right)^{1 / 2} \frac{d r}{c_{s}}+n_{p} \pi \approx \frac{\pi}{\Delta \nu}\left(\nu-\nu_{p}\right)+n_{p} \pi
$$

The phases $\epsilon_{g}\left(r_{a}, r_{b}, \omega\right)$ and $\epsilon_{p}\left(r_{c}, r_{d}, \omega\right)$ take into account the complex behavior close to the turning points and possible deviations from asymptotics. The phase $\epsilon_{g}$ was investigated theoretically by Takata et al. (2016a,b), Pincon et al. (2019) and observationally by Mosser et al. (2018) and Hekker et al. (2018). In a simplifying approach, Shibahashi (1979) neglected these complexities and disgarded the phases $\epsilon_{g}\left(r_{a}, r_{b}, \omega\right)$ and $\epsilon_{p}\left(r_{c}, r_{d}, \omega\right)$. 
Coupling factor: According to Shibahashi's approach, when the evanescent region is thick i.e. when $r_{c}-r_{b}>>\left(\int_{r_{c}}^{r_{d}}\left|k_{r}\right| d r\right)^{-1}$, the coupling factor is given to a good approximation by:

$$
q=\frac{1}{4} e^{-2 \theta_{e}} \approx \frac{1}{4}\left(\frac{r_{c}}{r_{b}}\right)^{-2}<<\frac{1}{4}
$$

where the phase for the evanescent region is given by:

$$
\theta_{e}=\int_{r_{b}}^{r_{c}}\left|k_{r}\right| d r
$$

In the other limit, the coupling factor for a thin evanescent region is given Takata $(2016 a, b)$ by:

$$
q=\frac{1-R}{1+R}
$$

where $R$ is the wave reflexion factor. For a thin evanescent region, $R<<1$ and $q \sim 1$.

For sake of simplicity in the following, we take $q$ independent of the frequency.

The resonant condition can take an alterative and equivalent form. Following Jiang \& ChristensenDalsgaard (2014) and Cunha et al (2015), Eq.9 is rewritten as

$$
\cos \theta_{g} \sin \theta_{p}-q \sin \theta_{g} \cos \theta_{p}=0
$$

which can be cast under the form

$$
C(\omega) \cos \left(\theta_{g}+\phi\right)=0
$$

where $C(\omega)$ and $\phi$ are defined such that $C(\omega) \cos \phi=\sin \theta_{p}$ and $C(\omega) \sin \phi=q \cos \theta_{p}$ and

$$
\phi=\tan ^{-1}\left(\frac{q}{\tan \theta_{p}}\right)
$$

From Eq.20 the resonant condition takes perhaps a more familiar form:

$$
\int_{r_{a}}^{r_{b}} k_{r} d r=\left(n_{g}+\frac{1}{2}\right) \pi-\phi
$$

i.e. this can be interpreted as the resonant condition for a g-mode perturbed by the p-mode cavity (represented by the phase $\phi-\pi / 2$ ).

The resonant condition Eq.9 was found to be amazingly useful to characterize mixed modes of post main sequence stars (see for instance Mosser et al. 2012, 2015, Deheuvels et al. 2012, 2014, 2015, Goupil et al 2013, Vrard et al. 2016, Mosser et al 2018). For a comprehensive review, see Hekker \& Christensen-Dalsgaard (2017).

An expression for the inertia ratio, $\zeta:$ Insights in the propagative properties of the modes can be obtained with an investigation of the behavior of the inertia ratio, $\zeta$ (Eq.7). Based on Shibahashi (1979)'s approach, an expression for the inertia ratio was derived (Goupil et al., 2013, Deheuvels et al. 2015, Mosser et al. 2015) as:

$$
\zeta(\nu)=\left(1+\frac{1}{q} \frac{\cos ^{2} \theta_{g}}{\cos ^{2} \theta_{p}} \frac{\Delta \Pi_{1} \nu^{2}}{\Delta \nu}\right)^{-1}
$$

where $\theta_{g}, \theta_{p}, \Delta \Pi_{1}, \nu_{p}$ and $\Delta \nu$ are defined in Eq.14, Eq.17, Eq.16 and Eq.1 respectively. 


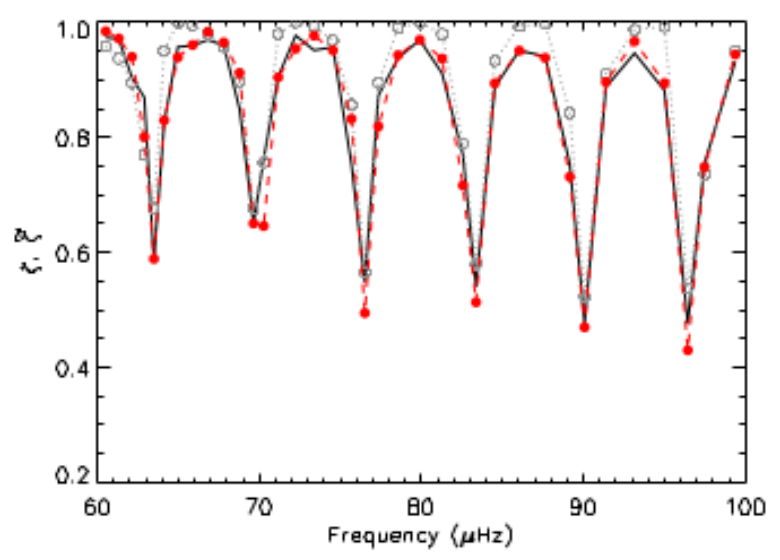

Figure 7: Inertia ratio $\zeta$ for $\ell=1$ modes of a model for the Kepler star KIC7581399 computed from the numerical eigenfunctions (black solid line) and from approximate analytical expressions with various degrees of approximation (red filled circles and red dashed line; gray dotted line and open circles) (credit Deheuvels et al 2015).

For a given stellar model, $\zeta(\nu)$ (Eq.7) can be computed using numerical eigenfunctions, solutions of the oscillation equations (Unno et al., 1989). The comparison with the analytical approximate formulation, Eq.23, shows a good agreement. The $\zeta$ variation from one mode to the next one is represented as a function of frequency in Fig.7 for a model for the Kepler star KIC7581399. Fig.7 shows that the values of the local maxima of $\zeta$ (associated with g-dominated modes) do not vary much with frequency whereas the local minima (associated to p-dominated modes) values decrease with increasing frequencies.

The local maxima and minima of $\zeta$ are given by (Mosser et al., 2015):

$$
\zeta_{\min } \approx\left(1+\frac{1}{q \mathcal{N}}\right)^{-1} ; \zeta_{\max } \approx\left(1+\frac{q}{\mathcal{N}}\right)^{-1}
$$

where

$$
\mathcal{N} \approx \frac{\Delta \nu}{\Delta \Pi_{1}} \frac{1}{\nu^{2}}=\frac{1}{\Delta \nu \Delta \Pi_{1}}\left(\frac{\Delta \nu}{\nu}\right)^{2}
$$

The coupling factor, $q$, takes values in the range $(0,1)$. The non dimensional quantity $1 /\left(\Delta \nu \Delta \Pi_{1}\right)$ corresponds to the number of mixed modes sitting between two consecutive p-dominated modes within a $\Delta \nu$ frequency interval. This quantity usually is much larger than 1. From Eq.25, we have:

$$
\frac{q}{\mathcal{N}} \approx q \Delta \nu \Delta \Pi_{1} n_{p}^{2} ; \frac{1}{q \mathcal{N}} \approx \frac{1}{q} \Delta \nu \Delta \Pi_{1} n_{p}^{2}
$$

where $n_{p}=\nu / \Delta \nu$. The factors $q \Delta \nu \Delta \Pi_{1}$ and $\Delta \nu \Delta \Pi_{1} / q$ are displayed in Fig.8 as a function of $\Delta \nu$ for the Mosser et al 2017's sample of stars. The factor $q \Delta \nu \Delta \Pi_{1}$ remains smaller than $4.10^{-3}$ for the RGB star sample of interest here. For RGB stars, these factors tend to increase with evolution represented by $\Delta \nu$ here. Further, for the detected modes in the vicinity of $\nu_{\max }$ corresponding to the maximum of the power spectrum, $n_{p} \sim 6-10$ for the sample of RGB stars studied by Mosser et al. (2017), Vrard et al. (2016). We then expect $\mathcal{N}>>1$ and $q / \mathcal{N}<<1$ which implies that $\zeta_{\max } \sim 1$.

From Eq.24, one derives the $\zeta$ ratio as:

$$
\frac{\zeta_{\min }}{\zeta_{\max }} \approx\left(1+\frac{1}{q \mathcal{N}}\right)^{-1}\left(1+\frac{q}{\mathcal{N}}\right) \sim \frac{1}{1+\frac{\Delta \nu \Delta \Pi_{1}}{q} n_{p}^{2}}
$$



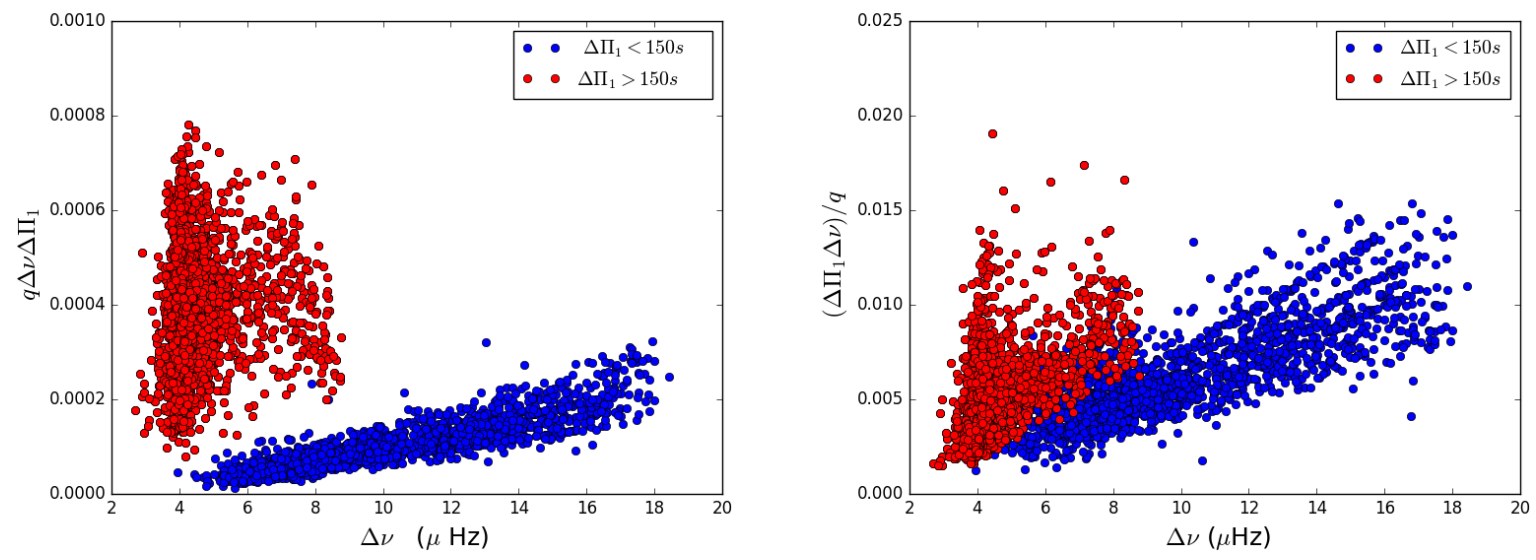

Figure 8: left: values of the factor $q \Delta \Pi_{1} \Delta \nu$ as a function of $\Delta \nu$ computed for the data set from Mosser et al. (2017). Stars with $\Delta \Pi_{1}<150$ roughly correspond to $R G B$ stars while stars with $\Delta \Pi_{1}>150$ s are clump stars. Right: same as left for the factor $\left(\Delta \Pi_{1} \Delta \nu\right) / q$

For RGB stars, this ratio tends to decrease with $n_{p}^{2}$ with a rate given by the ratio $q /\left(\Delta \nu \Delta \Pi_{1}\right)$.

As an illustration, I consider the RGB star, KIC 4448777, studied by M.P Di Mauro and collaborators (Di Mauro et al, 2018). Two best fitting models give the mass and radius of the star to be either $(1.0,3.94)$ or $(1.13,4.08)$ in solar units, respectively (Di Mauro et al, 2018) . Mosser et al. (2018) and Vrard et al. (2018) provided the values of the characteristic quantities for that star that-is $q=0.14 \pm 0.05, \Delta \nu=16.95-17.01 \mu \mathrm{Hz}, \Delta \Pi_{1}=89.3 \pm 1.76 \mathrm{~s}$. The radial order in the vicinity of $\nu_{\max }$ is $n_{p}=11$. With these values, one obtains: $q / \mathcal{N}=q \Delta \nu \Delta \Pi_{1} n_{p}^{2}=2.1210^{-4} n_{p}^{2}=$ $7.6510^{-3}-4.7810^{-2}<<1$ for $n_{p}$ in the range $(6,15)$ and $1 /(q \mathcal{N}) \sim 0.011 n_{p}^{2} \sim 0.39-2.44$ and $\zeta_{\min } / \zeta_{\max } \sim 0.44-0.0022\left(n_{p}^{2}-121\right)$.

Rotational splitting: The theoretical rotational splitting is defined as

$$
\delta \nu_{n, m}=m \int_{0}^{R} K_{n}(r) \frac{\Omega(r)}{2 \pi} d r
$$

where the angular dependence has been assumed averaged out to keep only the radial dependence. The kernel $K_{n}(r)$ involves the eigenfunction and structure of the star through the pressure and other profiles (Christensen-Dalsgaard, 2007).

Fig.9 shows the rotational splittings computed from Eq. 28 with the numerically computed eigenfunctions and assuming a rotation profile decreasing outward. The local maxima of the splittings are those associated with g-dominated modes while the local minima correspond to the p-dominated modes. Measurements of the splitting maximum provides the core rotation rate (Beck et al. 2012, Mosser et al. 2012b).

$$
\delta \nu_{\max } \approx \frac{\Omega_{\text {core }}}{4 \pi}
$$

Further Goupil et al. (2013) showed that the rotational splittings follow the behavior of the inertia ratio $\zeta$ (Fig.10). From Eq.15, Eq.A3 and Eq.A6 of that Goupil et al. (2013)'s paper, the splitting can be written as:

$$
\delta \nu \approx(1-\zeta) \frac{\Omega_{e n v}}{2 \pi}+\frac{\zeta}{2} \frac{\Omega_{\text {core }}}{2 \pi}
$$




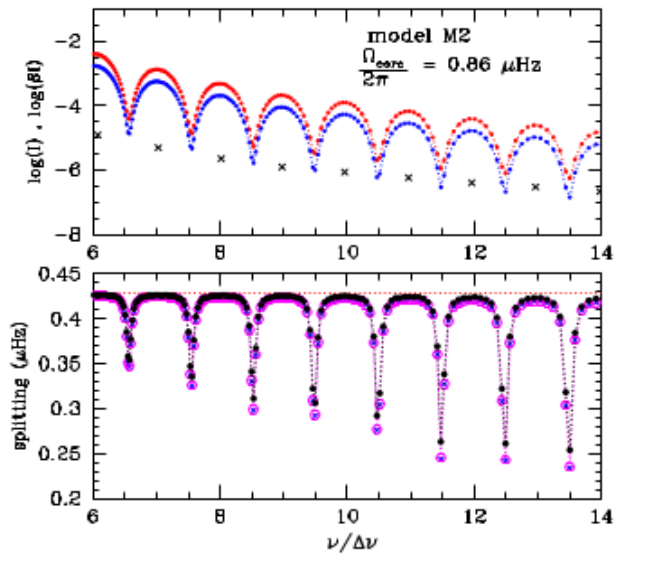

Figure 9: Top: inertia ratio as a function of frequency numerically computed for a $1.3 M_{\odot}$ red giant model. Crosses, red and blue dots represent inertia ratios for $\ell=0,1,2$ respectively. Bottom: theoretical rotational splitting as a function the normalized frequencies of (credit Goupil et al, 2013).

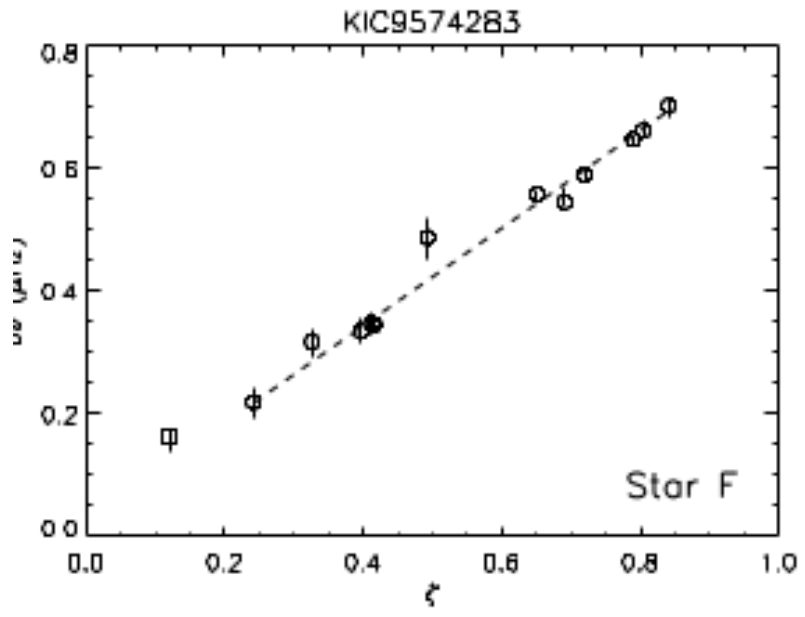

Figure 10: Observed rotational splittings as a function of the inertia ratio for the Kepler star KIC8751420. (circles for $\ell=1$ modes and squares for $\ell=2$ modes). The black dot-dashed line shows a linear regression of the observed splittings as a function of $\zeta$ (credit Deheuvels et al., 2014).

Then the local maxima are

$$
\delta \nu_{\max } \approx \frac{\zeta_{\max }}{2} \frac{\Omega_{\text {core }}}{2 \pi}\left(1+2 \frac{\left(1-\zeta_{\max }\right)}{\zeta_{\max }} \mathcal{R}\right) \approx \frac{1}{2} \frac{\Omega_{\text {core }}}{2 \pi} \frac{1+2(q / \mathcal{N}) \mathcal{R}}{1+q / \mathcal{N}}
$$

where Eq.24 was used and $\Omega_{e n v}$ and $\Omega_{\text {core }}$ represent seimic averages of the rotation rate over the core and over the envelope respectively; $\mathcal{R}=\Omega_{\text {env }} / \Omega_{\text {core }}$ is the rotation ratio or 'gradient'. Stellar models predict that $\mathcal{R}$ decreases when the star ascends the RGB. For the most g-dominated modes, $q / \mathcal{N}<<1$ then one recovers Eq.29. Actually, $\delta \nu_{\max }$ slightly decreases with frequency. Eq.31 well reproduces this decrease of the local maxima with the frequency. An example is shown in Fig.11.

Since individual $\delta \nu$ and $\zeta(\nu)$ are observable, one has access to a precise measurement of $\Omega_{\text {core }}$ (Eq.29 or Eq.31) and -provided that p dominated modes are detectable- to $\Omega_{\text {env }}$ or $\Omega_{\text {env }} / \Omega_{\text {core }}$ (Eq.30). This was validated by comparison with results of numerical models and observations.

As shown by Klion et al. (2017), the ratio $\mathcal{R}$ depends on the location of the differential rotation within the star. $\mathcal{R}$ can be obtained at a given frequency. Klion et al. (2017) suggested to use $\delta \nu_{\min } / \delta \nu_{\max }$ in the vicinity of $\nu_{\max }$, the frequency at maximum power, in order to obtain information about the location of the differential rotation. Setting $n_{p}$ to $n_{p, \max }=\nu_{\max } / \Delta \nu$, the splitting ratio then only depends on $\mathcal{R}=\Omega_{\text {env }} / \Omega_{\text {core }}$ and on the observable $\Delta \nu \Delta \Pi_{1} / q$ for each star. A plot of $\delta \nu_{\min } / \delta \nu_{\max }$ as a function of $\Delta \nu \Delta \Pi_{1} / q$ provides the rotation gradient $\mathcal{R}$. Following this idea, a general fit can also in theory provides the ratio $\mathcal{R}$. Using Eq.30 and Eq.24, one derives the following expression for the splitting ratio:

$$
\frac{\delta \nu_{\text {min }}}{\delta \nu_{\max }} \approx \frac{\left(1-\zeta_{\text {min }}\right) \Omega_{\text {env }}+\left(\zeta_{\text {min }} / 2\right) \Omega_{\text {core }}}{\left(1-\zeta_{\text {max }}\right) \Omega_{\text {env }}+\left(\zeta_{\text {max }} / 2\right) \Omega_{\text {core }}}=\left(\frac{1+\frac{q}{\mathcal{N}}}{1+\frac{1}{q \mathcal{N}}}\right)\left(\frac{1+2 \frac{1}{q \mathcal{N}} \mathcal{R}}{1+2 \frac{q}{\mathcal{N}} \mathcal{R}}\right)
$$

In Fig.11(left), the splitting ratio are plotted as a function of the radial order $n_{p}=\nu / \Delta \nu$ for several values of the rotation ratio $\mathcal{R}$. For large differential rotation $\mathcal{R}$, the splitting ratio increases with $n_{p}$ whereas for weak differential rotation $\mathcal{R}$, it decreases with $n_{p}$. Fig.11(right) displays the observed 

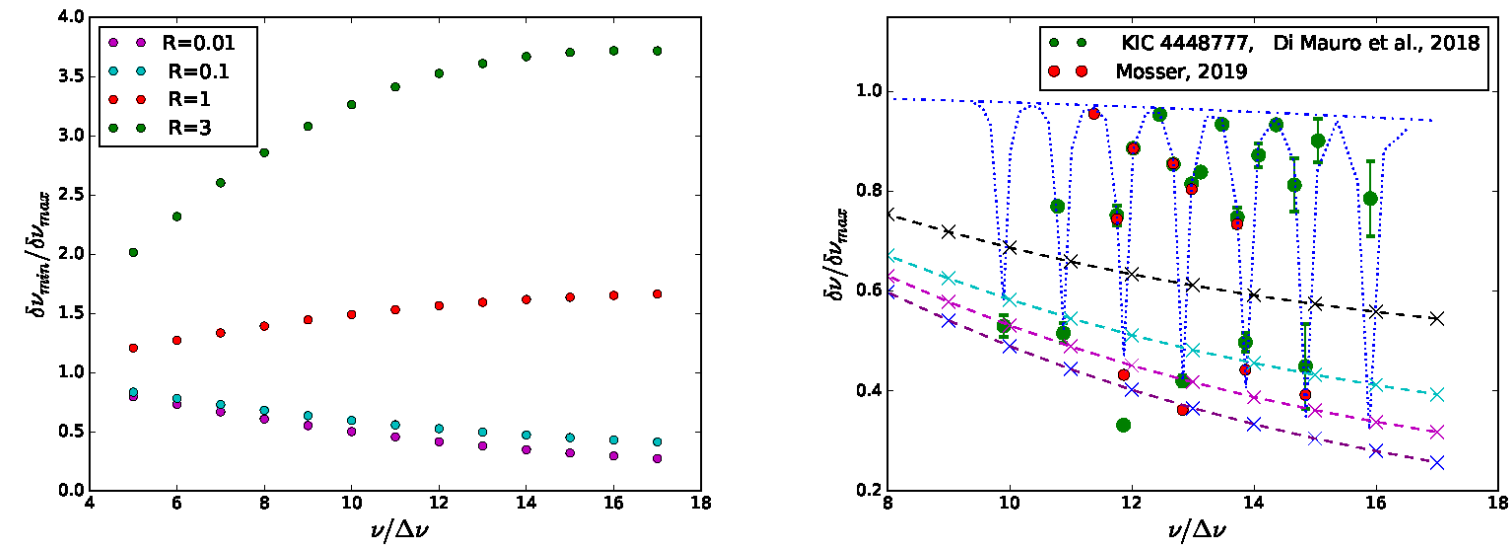

Figure 11: left: Splitting ratio as a function of the radial order $\nu / \Delta \nu$ for several values of the rotation ratio $\mathcal{R}$. Right: observed splittings for KIC 448777 normalized to the constant value $0.4 \mu \mathrm{Hz}$ as a function of $\nu / \Delta \nu$ . The dotted curve represents $\delta \nu_{\max } / 0.4=1 /(1+q \mathcal{N})$. Crosses connected with dashed lines show the discrete values of the ratios $(1+2 \mathcal{R} /(q \mathcal{N}) /(1+1 /(q \mathcal{N}))$ for $\mathcal{R}=0.2$ (black), $\mathcal{R}=0.1$ (cyan), $\mathcal{R}=0.05$ (magenta), $\mathcal{R}=0.01$ (purple).

splittings derived by Di Mauro et al. (2018) as well as those provided by my long-time colleague B. Mosser (Mosser, 2019, priv. comm.) for the star KIC4448777 discussed in the previous section. The splittings are normalized to the constant value $0.4 \mu \mathrm{Hz}$ (close to $\delta \nu_{\max } \sim \Omega_{\text {core }} / 4 \pi$ ) as a function of the radial order $n_{p}=\nu / \Delta \nu$. The pattern of splitting variation with frequency as obtained using an asymptotic fit to the observed values is also shown. These observations are compared with the theoretical curve $\delta \nu_{\max } / 0.4 \mu \mathrm{Hz}=1 /(1+q \mathcal{N})$ which reproduces well the observed slight decrease of $\delta \nu_{\max }$ with frequency. Comparison with the theoretical ratio

$$
\frac{\delta \nu_{\min }}{0.4 \mu H z} \sim \frac{1+2 \mathcal{R} /(q \mathcal{N})}{1+1 /(q \mathcal{N})}
$$

shows that it captures well the behavior of the local splitting minima as a function of $n_{p}$. It is plotted for various values of the rotation gradient $\mathcal{R}$. Below $\mathcal{R}<0.01$, the curves are superimposed, showing that no information can be obtained when the rotation gradient is too small. On the other extreme, values of $\mathcal{R}$ larger than 0.25 are clearly in disagreement with the observations for that star. A much more precise determination of the local minima appears to be necessary in order to obtain a real constraint on the location of the differential rotation. A more detailed study involving a large sample of RBG stars is in progress (Goupil and Mosser, 2020, in prep.).

Period spacing The period spacings $\Delta P_{n}=P_{n}-P_{n-1}$ numerically computed from a stellar model of an evolved 1.3 $M_{\odot}$ star are plotted as a function of the frequency in Fig.12. The same recurrent pattern is observed than for the inertia ratio and the rotational splittings. Actually deriving Eq.9 with respect to the radial order, Mosser et al. (2015) found that

$$
\frac{\Delta P}{\Delta \Pi_{1}} \sim \zeta(\nu)
$$

The validity of this relation can be established by comparing the numerical values of $\Delta P$ for a stellar model of red giant on one hand and $\zeta(\nu) \Delta \Pi_{1}$ on the other hand. Fig. 12 shows a perfect agreement when $\zeta$ is also computed numerically. When the asymptotic expression for $\zeta$ (Eq.23) is used, some deviations can occur because of some departures from the asymptotics. 


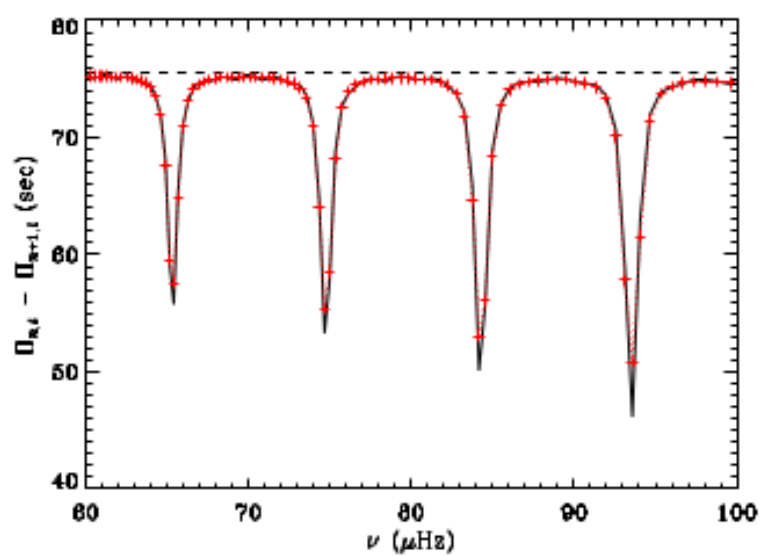

Figure 12: Numerical period spacings computed for $\ell=1$ modes for a $1.3 M_{\odot}$ red giant model as a function of frequency (the discrete values are continuously connected with the black solid lines). The dashed horizotal line represents the asymptotic period spacing. The red lines and symbols show the period spacings computed using by Eq.33 (credit Hekker et al., 2017).

With $\zeta(\nu)$ given by Eq.23, one obtains

$$
\frac{\Delta P}{\Delta \Pi_{1}}=\left(1+\frac{1}{q} \frac{\cos ^{2} \theta_{g}}{\cos ^{2} \theta_{p}} \frac{\Delta \Pi_{1} \nu^{2}}{\Delta \nu}\right)^{-1}
$$

An alternative but equivalent expression can be derived from Eq.20 (Jiang \& Christensen-Dalsgaard, 2014, Cunha et al., 2015). Eq.22 is rewritten as

$$
\frac{\omega_{g}}{\omega}+\phi=\pi(n+1 / 2)
$$

from which we compute

$$
\omega_{g}\left(P_{n+1}-P_{n}\right)+\phi_{n+1}-\phi_{n}=\pi
$$

and derive

$$
\omega_{g} \Delta P\left(1+\frac{1}{\omega_{g}} \frac{\Delta \phi}{\Delta \omega} \frac{\Delta \omega}{\Delta P}\right)=\pi
$$

which can easily be rewritten as:

$$
\frac{\Delta P}{\Delta \Pi_{1}}=\left(1-\frac{\omega^{2}}{2 \pi \omega_{g}} \frac{d \phi}{d \omega}\right)^{-1}
$$

From the expression of $\phi$, we obtain

$$
\frac{d \phi}{d \omega}=\frac{q}{\sin ^{2} \theta_{p}+q^{2} \cos ^{2} \theta_{p}} \frac{d \theta_{p}}{d \omega}=\frac{q}{\sin ^{2} \theta_{p}+q^{2} \cos ^{2} \theta_{p}} \frac{1}{\omega_{p}}
$$

then

$$
\frac{\Delta P}{\Delta \Pi_{1}}=\left(1-\frac{\omega^{2}}{2 \pi \omega_{g} \omega_{p}} \frac{q}{\sin ^{2} \theta_{p}+q^{2} \cos ^{2} \theta_{p}}\right)^{-1}=\left(1-\frac{\nu^{2}}{2 \pi \nu_{g} \nu_{p}} \frac{1}{q \cos ^{2} \theta_{p}} \cos ^{2} \theta_{g}\right)^{-1}
$$

where we have used $d \theta_{p} / d \omega \approx 1 / \omega_{p}$ and Eq.9.

The highly precised $\Delta \Pi_{1}$ values led to tight constraints about the $N$ profile averaged over the core hence on the physical mechanisms that shape $N$ in the central region as discussed in the next section. 


\section{Mixing beyond the convective core of red clump stars}

A major difficulty in the modelling of the central regions of red clump stars is the determination of the location of the convective core boundary and of the properties (thermal and chemical stratifications) of the medium in its vicinity. Stellar evolution codes use a local prescription to locate convectively unstable regions (basically the Schwarzschild criterion in the mixing length theory (MLT) framework or some improved variant formulation). The location usually is established by the change of sign of the temperature gradient difference $\nabla_{r a d}-\nabla_{a d}$ : convectively stable regions verify $\nabla_{r a d}<\nabla_{a d}$ whereas they are convectively unstable otherwise. Here $\nabla_{\text {rad }}$ represents the temperature gradient as if all the flux were transported by radiation and $\nabla_{a d}$ is the temperature gradient of an adiabatically stratified fluid. For convenience, the temperature gradient is defined with respect to the pressure $P$ i.e. $\nabla=d \log T / d \log P$. The central region identified as convectively unstable defines the formal convective core (hereafter FCC). This region is then assumed instantaneously chemically mixed and its thermal stratification assumed adiabatic. Generally speaking however, one expects that the fluid overshoots beyond the FCC into adjacent convectively stable regions where the fluid motion is braked down to a stop over some penetration distance. In that region, partial to full chemical mixing must occur and the thermal stratification can be modified. This then rises several questions:

- how setting up properly the convective core boundary and its evolution with time?

- how far does the extra -mixing region extend?

- does the thermal stratification become adiabatic beyond the FCC limit?

- is the chemical composition fully or partially mixed in the penetration region?

- in case of partial mixing, what is the profile chemical gradient? How does it evolve?

The evolution of the central layers of stars with a convective core has been a longstanding problem over several decades (see the review by Salaris \& Cassisi, 2017, see also Noels et al., 2010, Arnett et al. 2015, 2018, Paxton et al. 2018 and references therein). Various options for describing the region above the FCC can be found in the litterature: overshooting with instantaneous mixing or overshoot with time-dependent diffusive mixing. According to Zahn's 1991 terminology, those options refer to the case when the fluid moves into the stable layers but does not modify the thermal stratification, hence the temperature gradient remains radiative. Convective penetration instead refers to the case when energy transfer is efficient enough that the thermal stratification becomes adiabatic in the overshoot region. In addition, in a convectively unstable layer according to the Schwarzschilds's criterion where a chemical composition gradient exists and stabilizes the medium (Ledoux's criterion), mixing refered to as semiconvection can occur (Noels, 2013). The consequences of these various options translate into large uncertainties for the further evolution and structural changes of such stars.

A first clarification was given by Gabriel et al. (2014) based on earlier works. The convective boundary must be located at the layer where the fluid velocity vanishes in the framework of the MLT, the location of convective boundaries must be strictly set at the layer where $\nabla_{r a d}=\nabla_{a d}$ (convective neutrality). Its numerical implementation must be established from the convective side of the region, this is particularly crucial when a chemical discontinuity and/or gradient exists across the convective boundary. This is the case for red clump stars. Search for convective neutrality in presence of a discontinuous chemical composition across the convective core boundary and/or a gradient of chemical composition in the adjacent radiative region automatically leads to include an instantaneous mixing, mimicking fully mixed convective penetration (independently of the physical origin of the mixing). The situation is even further complicated for red clump stars because the burning of helium into 


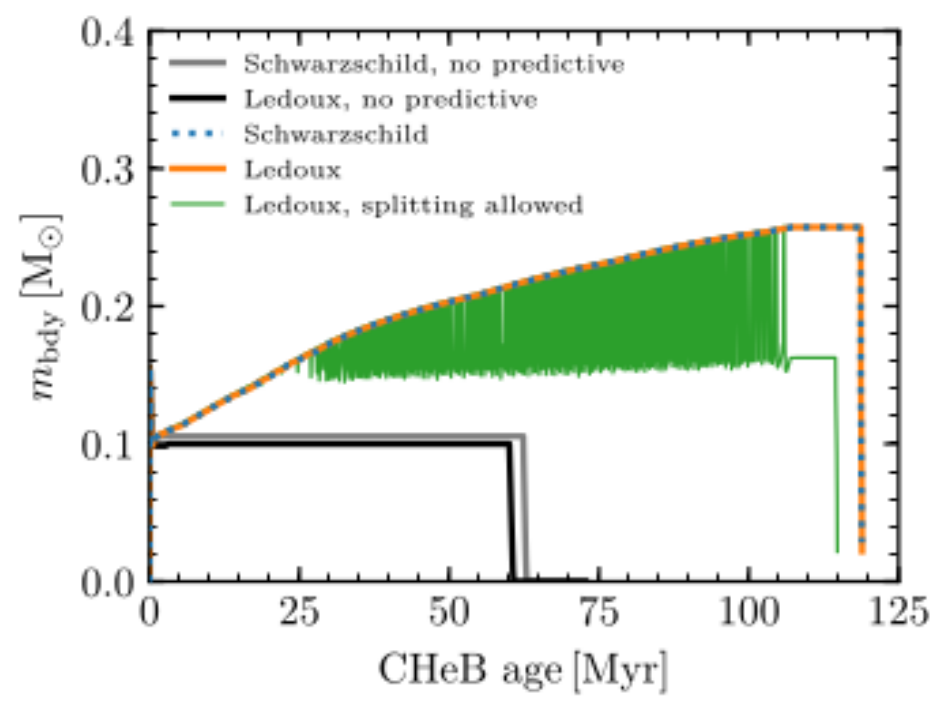

Figure 13: Evolution of the mass of the convective core of a $1 M_{\odot}$ model with various mixing and numerical scheme options. The FCC core (grey line) for instance does not grow in mass with time (credit Paxton et al, 2019).

carbon and oxygen generates a local increase of the opacity within the convective region which can decrease the radiative gradient there and can eventually cause a split of the convective region into two convective layers separated by a semiconvective region. The modelling of the thermal and chemical stratifications (full or partial mixing, adiabatic stratification or radiative one) in such regions therefore remains highly uncertain. In any case, a proper implementation of the Schwarzschild criterion and all formulations with extra-mixing lead to larger (more massive) convective core than FCC. Depending on the values of the adopted parameters in these empirical formulations and on the adopted formulation itself, the convective cores have different sizes and different chemical profiles develop in the adjacent overlying regions (Fig.13). Several numerical schema are investigated to overcome this issue (Paxton et al., 2019 and references therein).

Clearly, observational constraints about the central properties of these stars can help making significant advances in that field. Montalban et al. (2013) and Montalban \& Noels (2014) indeed showed that the period spacing Eq. 4 is a sensitive probe of the properties in the central part of red clump stars. Fig.14- taken from Constantino et al. (2015)- illustrates the impact on the Brünt -Vaissala profile of various extra-mixing schemes. A modification of the Brünt-Vaissala profile has a direct impact on the period spacing (Eq.4) and therefore offers a possibility to be detected observationnally. In Fig.6 the increase of the mean period spacing when including overshooting compared to assuming a mere FCC is clearly seen.

The seismic observations of red giants and more specifically red clump stars provided by the space mission CoRoT and Kepler are in that sense invaluable. Montalban et al. (2013) compared in a $\Delta \Pi_{1}-\Delta \nu$ diagram the observed period spacings of red giant stars derived by Mosser et al. (2012a) to the theoretical asymptotic counterpart $\Delta \Pi_{1}$ computed from stellar models. The comparison requires some care but the authors found that stellar models of red clump stars built with the FCC option provide period spacings significantly smaller (i.e. by about $20 \%$ ) than the observed ones (Fig.15). The authors showed that adding an extra mixing (overshoot from He burning core) can help decreasing the discrepancy as it pushes outward the inner boundary of the g-mode cavity hence increases $\Delta \Pi_{o b s}$ by $20 \%$ (a discrepancy of the order of $30 \mathrm{~s}$ ). For instance the period spacing increases from $\Delta \Pi=255 s$ up to $\Delta \Pi=305 s$ for a stellar model with $1.5 M_{\odot}$ in the middle of the He burning phase. 


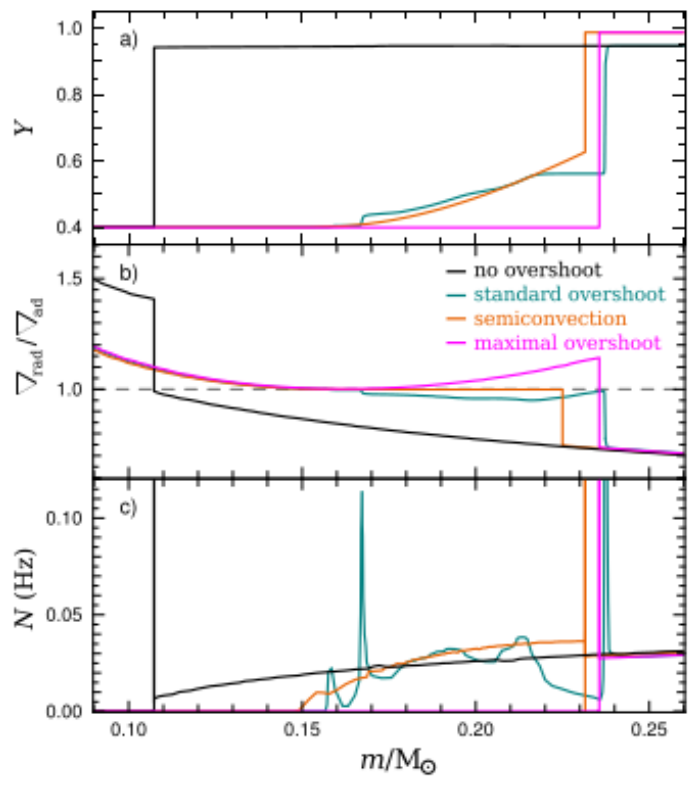

Figure 14: Helium profile (top), $\nabla_{\text {rad }} / \nabla_{\text {ad }}$ profile (middle) and $N$ profile (bottom) for a $1.5 M_{\odot}$ model at the evolutionary phase where $60 \%$ of helium was burned. Four different schemes for core overshoot in a CheB star give rise to four different profiles of $N$ (Credit Constantino et al., 2015).

Bossini et al. (2015) and Constantino et al. (2015) further tested the impact of several options of mixing beyond the FCC using the period spacings derived for a set of Kepler red clump stars (Mosser 2012, 2014). Fig.16 taken from Constantino et al. (2015) clearly indicates that without any extra mixing beyond the FCC, the period spacings never reach the observed maximal values in a $\Delta \Pi_{1}-\Delta \nu$ diagram. They agree with the conclusion that the chemically mixed core must be significantly larger than the FCC core as it can be seen in Fig.16 for instance. This confirms results from theoretical studies and numerical simulations and confrontation using classical (non-seismic) observations.

Interpretation of the $\Delta \Pi_{1}-\Delta \nu$ for field red clump stars may be complicated by their somewhat different metallicities. In order to avoid this complication, Bossini et al. (2017) studied red clumps stars belonging to two open clusters NGC 6791 (with representative stellar models $1.15 M_{\odot}$ with initial $Z=0.035$ and $Y=0.3$ ) and NGC 6819 (with representative stellar models $M=1.60 M_{\odot}$ with initial $Z=0.0176$, and $Y=0.267$ ). The seismic data were obtained by Vrard et al. (2016). The authors compared the behavior of $\Delta \Pi_{1}$ as a function of $\Delta \nu$ for stellar models including different modelling of the regions beyond the FCC. The results are summarized in Fig.17 (Fig.1 in Bossini et al., 2017). At a given age and metallicity, the convective core evolution causes an increase of $\Delta \Pi_{1}$ with $\Delta \nu$. The minimal value of $\Delta \Pi_{1}$ corresponds to less massive, young stars (beginning of the CheB phase) while the maximal value corresponds to more massive- hence slightly more evolvedstars (end of $\mathrm{CheB}$ phase). The comparison tends to favor the scheme where the thermal stratification in the extra-mixing region remains radiative at least at the beginning of the $\mathrm{CHeB}$ phase. The other option (PC) seems more in agreement with the three low mass stars in NGC6819. The result in that last case remains doubtful because the stars are more massive and the correct period spacings are more difficult to determine. For the late $\mathrm{CHeb}$ phase (most massive stars in the samples), the convection tends to split as discussed above. Therefore the value of the period spacing strongly depends on the adopted scheme for the region in between the two split parts but in any case, the assumption of the largest cores seem to be ruled out.

These interesting studies show that advances must come from larger samples of stars with given mass and metallicity. On the theoretical side, improvement of modelling during the late part of the 


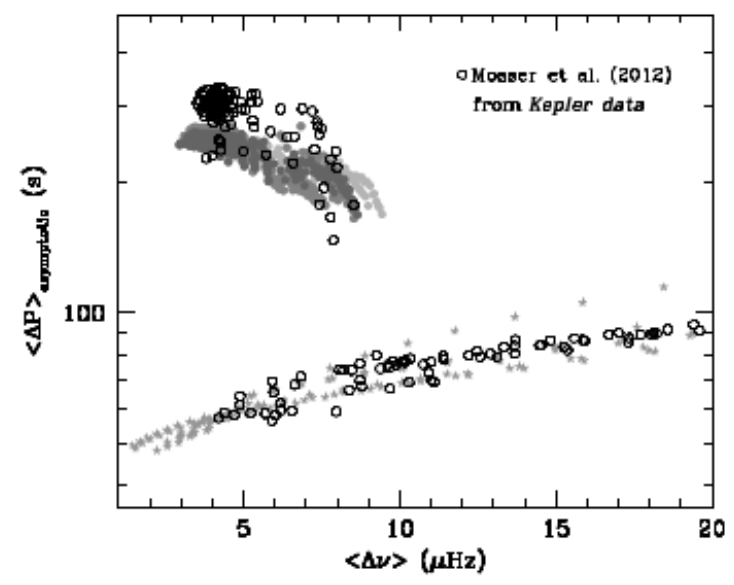

Figure 15: Period spacings as a a function of frequency for a model of red clump (top, left samples) and RGB stars (bottom samples). Filled dots: asymptotic period spacings from stellar models; open dots: observed period spacings from Mosser et al. (2012a) (credit Montalban et al. 2013)

CheB phase when the helium content in the burning region gets small - and the size of the core crucially depends on the modelling of the extra mixing beyond the FCC- are particularly necessary (Paxton et al., 2018, 2019 and references therein, Spruit, 2015, Constantino et al., 2017).

\section{Angular momentum transport in low mass post main sequence stars}

\subsection{Rotation profiles of red giant stars}

In 1D stellar modelling, the convective envelope of cool evolved stars is usually assumed to rotate uniformly ${ }^{1}$. If local conservation of angular momentum is maintained within the adjacent radiative region below, the core spins up and the envelope decelerates due to the contraction of the core and expansion of the envelope during the subgiant and giant phases. In that framework, cool, evolved stars are expected to develope a strong rotation gradient between the fastly rotating core and the slowly rotating envelope.

On the observational side, a lower limit of the surface rotation rate for subgiant and red giant stars, $\Omega_{\text {surf }}$, can be derived from spectroscopic measurements of the projected surface rotation $v \sin i$. The surface rotation period can also be obtained from photometric measurements of spot modulations when the star undergoes some type of surface activity. However because the surfaces of subgiants and red giants are mostly rotating slowly, the measurements are only available for a small subsample of stars biased toward the rapid rotators (Tayar et al., 2015; Ceillier et al., 2017). On the other hand, seismic measurements of the mean rotation rates of the core of evolved stars, $\Omega_{\text {core }}$, can be obtained from the maximum value reached by the rotational splittings $\delta \nu_{\max }$ (Eq.28) or from inversion methods using individual splittings (Fig.21). They are found of the order of a few ten days for subgiants and RGB stars and of some hundreds days for red clump stars (Beck et al., 2012; Mosser et al., 2012b, Deheuvels et al., 2012, 2014, 2015, Di Mauro et al. 2016, 2018). They actually are in good agreement with the rotation of the hot B subdwarf ( $\mathrm{sdB}$ ) stars which might be their descendants (Charpinet et al., 2018). The observed core rotation rates of subgiant and red giant stars are however much smaller than

\footnotetext{
${ }^{1}$ although this may not be the reality, see Brun \& Palacios 2009
} 


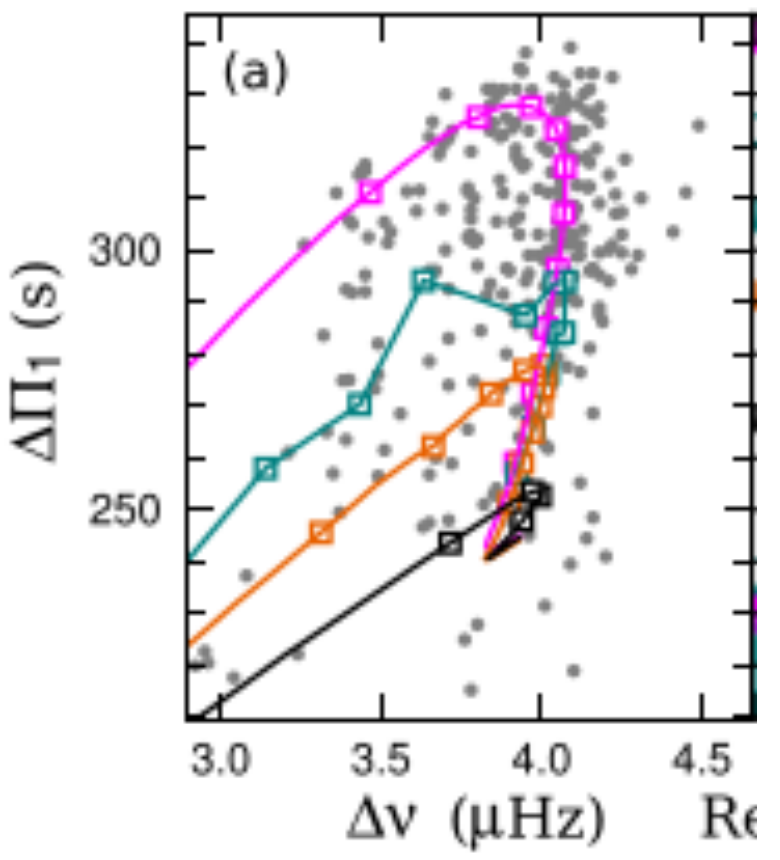

Figure 16: Period spacing as a a function of the large separation for stars in the red clump. Grey dots : observations from Mosser et al. $(2012,2014)$. Color lines corresponds to the evolution of a $1 M_{\odot}$ stellar models with several options for the modelling of extra mixing beyond the FCC (same color than in Fig.14) (Constantino et al. 2015).

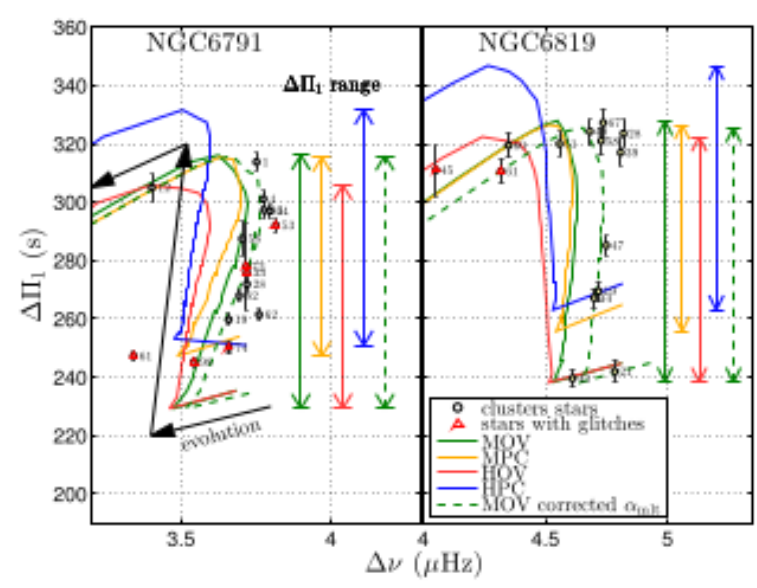

Figure 17: Period spacing as a function of the large separation for red clump stars in two open clusters (dots). Open symbols connected with lines correspond to period spacings for stellar models with mild overshoot (green), mild convective penetration (orange), high overshoot (red), blue (high convective penetration). The arrows delimite the range $\Delta \Pi_{1, \max }-\Delta \Pi_{1, \min }$ (Bossini et al. 2017).

what can be expected when assuming instantaneous angular momentum (hereafter AM) transport in convective regions and local conservation of angular momentum in radiative regions

Further, individual studies for six subgiants indicate that the star core rotation accelerates when evolving toward the RGB (Deheuvels et al., 2014) whereas a statistical study of 300 giants including 85 RGB stars showed that the core rotation of RGB stars seems to slow down when the star ascends the RGB (Mosser et al., 2012b). This is summarized in Fig.18 where the core rotation is plotted as a function of the radius (proxy for evolution). Such evolution of the core rotation cannot be reproduced by standard evolution models which do not include an efficient AM transport in the radiative interior of these cool stars. However the above view has been recently challenged : are the RGB cores really slowing down when ascending the giant branch? With a more sophisticated data analysis process, Gehan et al. (2018) obtained the core rotation rates for a much larger sample of red giant stars. The stellar radius is usually used as a proxy for the age of the star on the ascending red giant branch. Fig.19 reveals that despite a high dispersion, the spin down of the core rotation rate of the RGB stars seems to be weaker than previously established by Mosser et al. (2012). Further, Gehan et al. (2018) emphasized that the star does not enter the RGB phase with the same radius depending on its mass. The authors then argue that a better age indicator is the averaged number of g-dominated modes existing between two p-dominated modes, $\mathcal{N}$. With that indicator as a proxy for the age, the core does not seem to slow down when the stars ascends the giant branch (Fig.19) and this is found to hold true independenly of the mass of the star.

An additional constraint is given by the seismic measurements of the core-to-envelope rotation ratio $\Omega_{\text {core }} / \Omega_{\text {env }}$ which can be obtained in favorable cases as mentionned in Sect.2. Fig.18, right, illustrates the case when both envelope and core rotation rates are seismically determined for six 

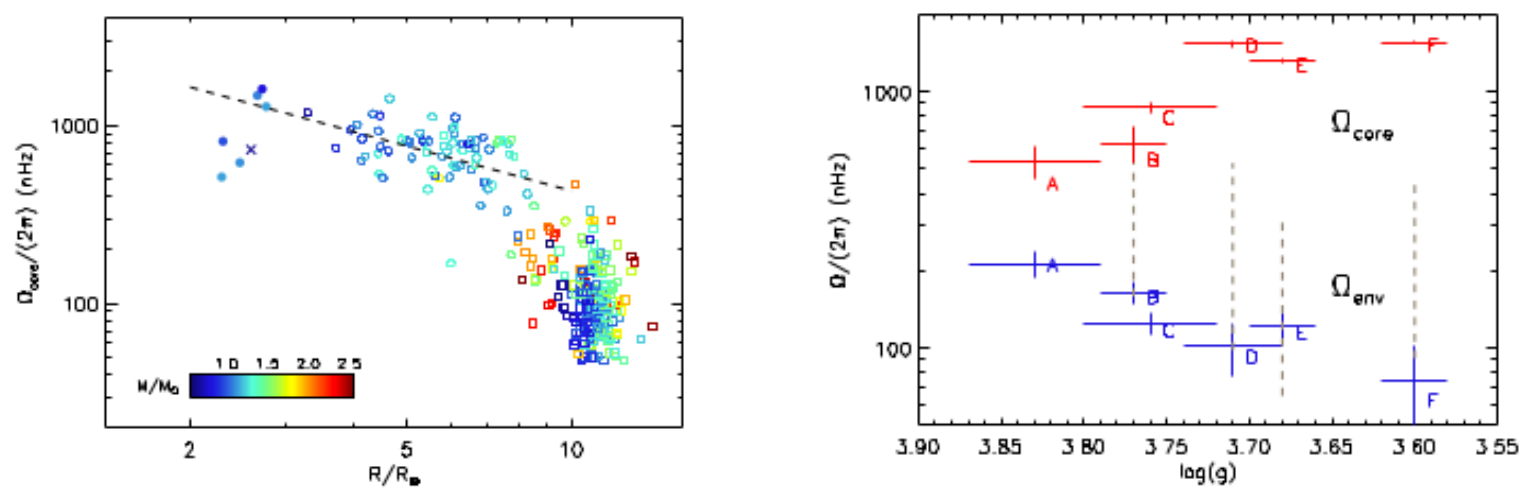

Figure 18: left: seismically measured core rotation of subgiants and red giants as a function of the seismically determined stellar radius. Evolution goes with increasing stellar radius. Stellar radii in the range 2-8 $R_{\odot}$ correspond to RGB stars. Clump stars have stellar radii larger than $8 R_{\odot}$. Right: Core and envelope seismic rotation rates for six subgiant stars and one red giant (Credit Deheuvels et al., 2014).
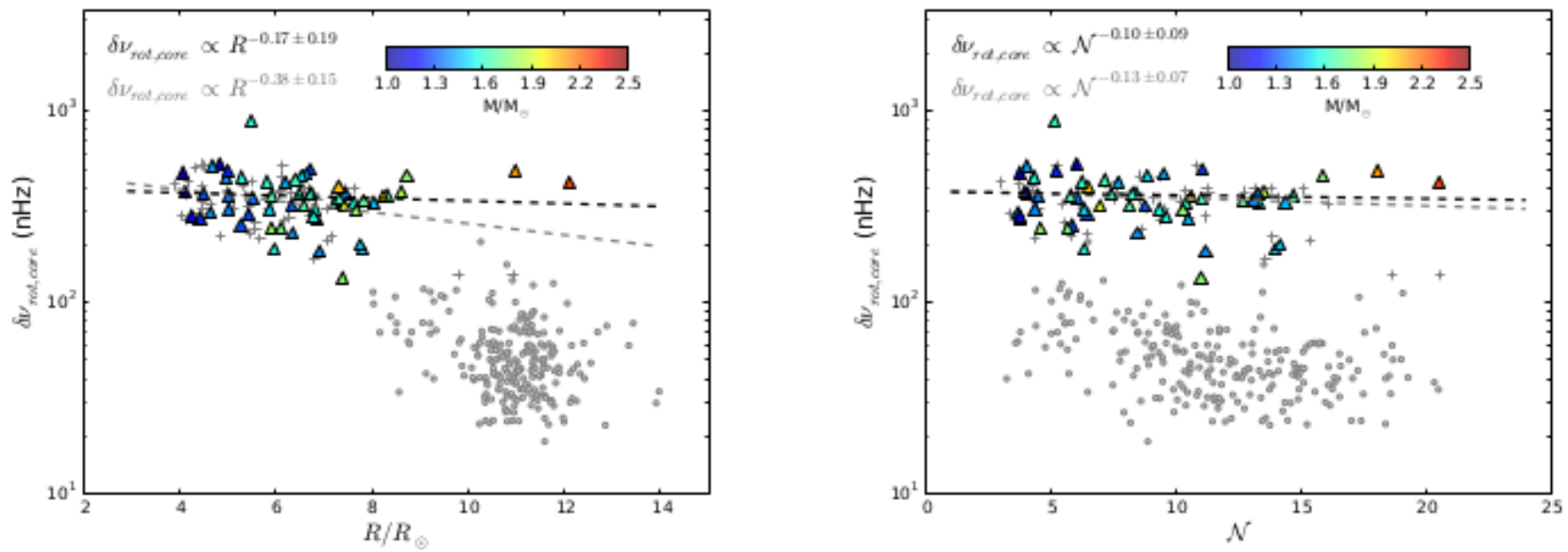

Figure 19: left: core contribution to the rotational splitting as a function of the stellar radius used as a proxy for age. Coloured symbols are for data from Gehan et al. (2008). Mosser et al(2012b)'s measurements on the $R G B$ and on the clump are represented by grey crosses and dots, respectively. Right: same as left but with the mode density $\mathcal{N}$ as a proxy for age (Credit Gehan et al., 2018).

subgiant stars (Deheuvels et al., 2014). Theses estimates indicate that the ratio $\Omega_{\text {core }} / \Omega_{\text {env }}$ lies in the range 2.5-20 and increases with evolution. For more evolved red giants, the contribution from the envelope to the rotational splittings becomes negligible and reliable estimates of $\Omega_{e n v}$ can no longer be obtained from the linear relation Eq.30.

The observations thus reveal that the inner cores of the PoMS stars rotate significantly faster than the envelopes albeit much less faster than predicted by standard stellar models when local conservation of AM is assumed in radiative regions. One therefore must admit that an efficient AM transfer is at work from the inner to the outer layers of these stars to compensate for the core spin up due to the contraction of the central layers beyond the MS phase. So the issues are:

- what mechanism(s) are able to slow down the core of red giants with evolution and reduce(s) the the radial differential rotation of evolved stars compared to what would be expected from evolution with local conservation of AM ?

- if several processes are at play, what is the dominant one?

- can these mechanisms be modelled properly enough in 1D stellar models to account for the 

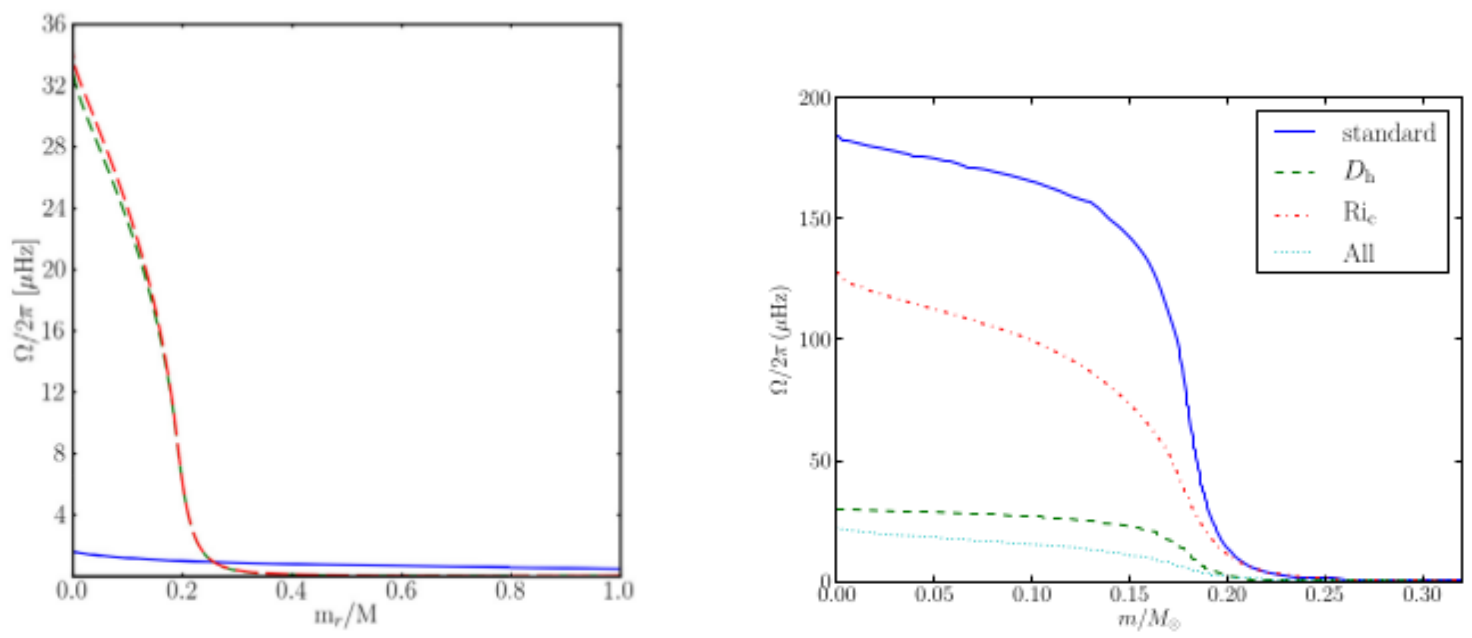

Figure 20: Left: Rotation profiles for $0.84 M_{\odot}$ models of the early red giant star KIC 7341231 at an age of 13.40 Gyr obtained with several assumptions about AM transport in the radiative region (black: assuming shellular rotation AM transport; blue : assuming uniform rotation on the main sequence, red : conservation of angular momentum during the subgiant phase : same as blue but $X_{c}=0$ ). The observed rotation range is $710 \pm 51 \mathrm{nHz}$ (Deheuvelset al., 2012) at the very bottom of the plot (Credit Ceillier et al., 2013). Right: Rotation profiles for $1.3 M_{\odot}$ models at the base of the $R G B$ when $R=3.73 R_{\odot}$ calculated assuming standard viscosity coefficients (label 'standard'), a vertical turbulent viscosity $D_{V}$ computed with the Richardson number $R i_{c}=1$ $\left(R i_{c}\right)$, a horizontal viscosity coefficient 100 times the standard value $\left(D_{h}\right)$, and combining all (All) (Credit Marques et al., 2013

above observations?

\subsection{What mechanism(s) can slow down enough the core of post-main-sequence stars?}

Several mechanisms of AM transport in radiative interiors can be invoked (Talon, 2008; Maeder 2009).

AM transport by hydrodynamical processes: At the basic level, structural changes due to evolution naturally generate differential rotation in radiative regions. This rotational shear can drive hydrodynamical instabilities leading to turbulence and therefore turbulent AM transport (Talon 2008). Historically the AM transport in radiative zones of 1D stellar models was then modelled as a pure diffusive process originating from the turbulence induced by a rotational shear. In that case, the AM vertical turbulent diffusion coefficient is parametrized and calibrated on observations (Endal \& Sofia 1978, 1981; Pinsonneault et al. (1989); this is still the case in several evolutionary codes. However with studies started more than 20 years ago, Zahn (1992) and co-workers showed that AM can be advected by large scale meridional motions in radiative regions of rotating stars, enhancing the rotational shear and competing with turbulent diffusive AM transport. This circulation is sustained by structural changes due to evolution, turbulence and by AM loss at the surface for low mass stars with convective envelopes. The rotation profile in radiative stellar regions of rotating stars therefore results from an advective-diffusive AM transport on secular time-scales (Zahn, 1992; Talon \& Zahn, 1997; Maeder \& Zahn 1998; Mathis \& Zahn 2004; Zahn 2013; see Palacios 2013 for a review).

While the assumption of a rotation rate a function of radius only, $\Omega=\Omega(r)$, was considered from the start in $1 \mathrm{D}$ stellar modelling, Zahn (1992) justifies this so-called shellular rotation as the result of 
a strongly anisotropic turbulence which causes a rapid homogeneization on horizontal surfaces. This assumption allows to reduce the AM transport description to a one dimensional process that-is in the vertical (i.e. radial) direction. In radiative regions, the local specific AM, $j(r)=r^{2} \Omega(r)$ then obeys a time-dependent equation of the form:

$$
\frac{\partial r^{2} \Omega}{\partial t}=-\dot{r} \frac{\partial r^{2} \Omega}{\partial r}-\frac{1}{\rho r^{2}} \frac{\partial \rho r^{2} \mathcal{F}_{t o t}}{\partial r}+\dot{j}_{\text {wind }}
$$

where the momentum flux is given by

$$
\mathcal{F}_{\text {tot }}=\mathcal{F}_{\text {circ }}+\mathcal{F}_{\text {shear }}
$$

and

$$
\mathcal{F}_{\text {circ }}=-\frac{1}{5} r^{2} \Omega U_{2} \quad ; \quad \mathcal{F}_{\text {shear }}=-r^{2} \Omega \nu_{v} \frac{\partial \ln \Omega}{\partial r}
$$

where $r(m)$ is the radius enclosing the mass, $\dot{r}$ is the time derivative of the radius, $\dot{j}_{\text {wind }}$ stands for the AM loss by a surface magnetized wind. The effect of the wind is to decelerate the rotation of the convective envelope, which is assumed to rotate rigidly at the same angular velocity than the surface. AM loss by magnetized wind is a complex 3D process, its modelling keeps on being improved and its impact on the rotation profile evolution being studied (Amard et al., 2016 and references therein). Equations and prescriptions for the vertical meridional circulation speed, $U_{2}$, and the vertical diffusion coefficient for vertical transport induced by a vertical shear, $\nu_{v}=\nu_{\text {shear }}$, entering Eq.34-36 above were established by Zahn (1992), Talon \& Zahn (1997), Maeder \& Zahn (1998), and Mathis \& Zahn (2004). The above equation is coupled to the evolution equations for the chemical elements not given here (Talon 2008, Maeder, 2009).

Advective-diffusive AM transport is implemented nowadays in several evolution codes but as already mentionned, the resulting AM transport appears to be insufficient to slow down enough the core rotation of red giant stars (Fig.20), a discrepancy which reaches one to two orders of magnitude depending on the star and the physical assumptions in the AM transport along the evolution (Eggenberger et al. 2012; Marques et al., 2013; Ceillier et al., 2013). While the advective-diffusive approach and the shellular approximation may still be reasonable for low mass, slowly rotating stars, several uncertainties remain in the modelling of the AM transport by hydrodynamical processes in stars. In particular the turbulent diffusion coefficients entering the equation for AM and chemical transports are mostly empirical (Meynet et al., 2013) and are currently being revised on more physical ground (Maeder et al 2013, Mathis et al., 2018).

In an attempt to render count of the rotation profiles of evolved low mass stars, the attention then turned to other transport processes as likely good candidates: AM transport by gravity waves, $\mathcal{F}_{\text {waves }}$ and normal modes $\mathcal{F}_{\text {modes }}$ and AM transport induced by magnetic instabilities.

Transport driven by internal gravity waves (IGW) $\mathcal{F}_{\text {waves }}$ : IGW are convectively excited in or near the base of the convective envelope and propagate in the radiative interior where they are eventually dissipated. The net AM transport results from the opposite contributions of retrograde and progade IGW. Assuming shellular rotation and core rotating faster than the enveloppe, the differential rotation serves as en efficient filter for the waves: only retrograde waves can propagate deep down toward the core and dissipate there while the prograde waves are rapidly damped close to the convective bottom of the envelope (Press, 1981; Schatzman, 1993; Zahn et al., 1997; for reviews, see Talon 2008; Mathis, 2013; Mathis \& Alfvan 2013). This asymetric propagation can decelerate the core if it occurs on time scales shorter than the evolutionary time scale. The IGW time scale strongly depends on the radiative dissipation. The radiative damping of the waves increases downward, therefore IGW 


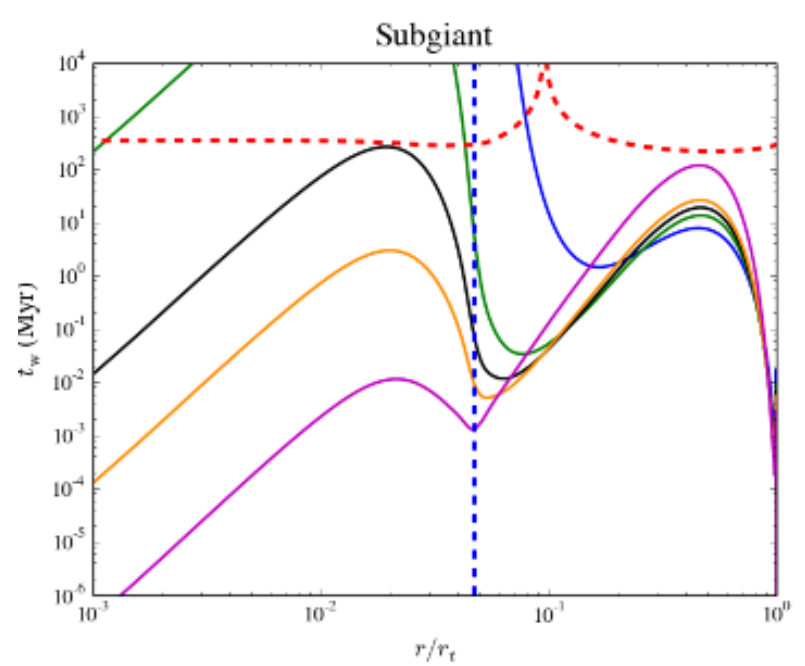

Figure 21: IGW time-scales (solid lines) as a function of the normalized radius in the radiative zone of a $1 M_{\odot}$ subgiant model. Colors correspond to different values for the amplitude of the differential rotation. The red and the blue dashed lines represent the contraction or dilatation timescale and the location of the hydrogen-burning shell, respectively. (Credit Pincon et al. 2017).

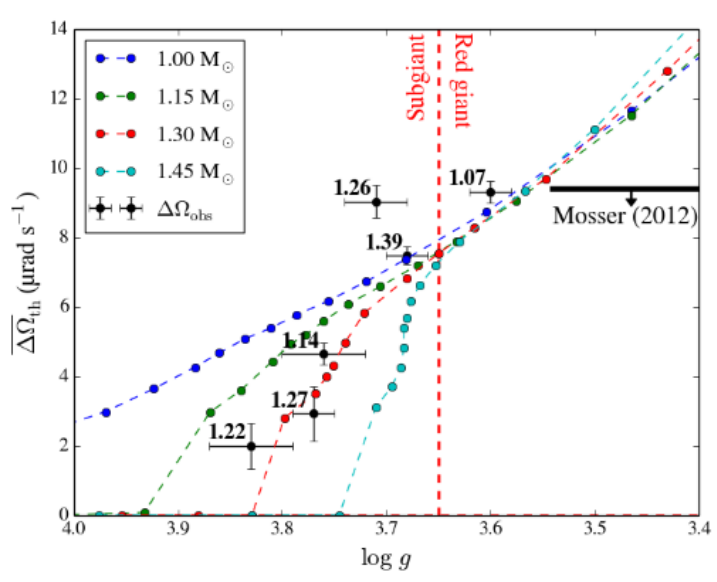

Figure 22: Evolution of the theoretical threshold for the amplitude of the radial-differential rotation $\Delta \bar{\Omega}$ resulting from IGW AM transport as a function of the stellar evolution proxy $\log g$. The vertical red dashed line marks the transition between the subgiant phase to the beginning of the RGB. The observed rotation contrasts $\Omega_{\text {core }}-\Omega_{\text {surf for the }}$ subgiants are derived from Deheuvels et al. 2014 and shown as error bars. The horizontal thick black line indicates the observed maximum amplitude of the differential amplitude on the $R G B$ as derived by Mosser et al. (2012) (Credit Pincon et al. 2017).

must be excited to sufficient amplitudes at the bottom of the convective envelope in order to reach the core and extract sufficient AM there. The IGW time scale therefore also depends on the wave excitation process at the base of the convective envelope and is one of the main uncertainties of this AM transport process.

Two types of excitation mechanisms have so far been studied for post main sequence stars: the IGW excitation by turbulent convection is not efficient enough for the IGWs to overcome the strong dissipation at the $\mathrm{H}$ shell burning neither during the subgiant phase nor later during the giant phase. It cannot therefore explain the observed subgiants and RG core rotations (Talon \& Charbonnel, 2008; Lecoanet \& Quataert, 2013; Fuller et al. 2014; Pincon et al., 2016). On the other hand, IGW excitation triggered by the penetration of turbulent plumes overshooting the radiative region below the $\mathrm{CZ}$ generates an AM transport on times scales that can be shorter than the evolutionary time scale (Fig.21, Pincon et al. 2017). With this type of excitation, IGW are able to slow down the subgiant core rotation when the differential rotation between the core and the bottom of the envelope is large enough to decrease the dissipation of the retrograde waves. Pincon et al. (2017) suggest that a self regulating process imposes the rotation rate to be close to the rotation threshold which seems in agreement with observations (Fig.22). Detailed computations of the evolution of the rotation profile of a low mass star including both IGW excitation mechanisms remain to be done. It must also be stressed that the IGW generation and propagation suffer from additional complexities such as critical layers (Alvan et al., 2013), the impact of rotation (Andre et al., 2018) and magnetic field (Loi \& Papaloizou, 2018) that are being investigated in current theoretical and numerical works. The net result of IGW is the propagation of successive fronts from the center to the surface. The internal rotation rate is then expected to be non uniform and the location of the rotation maximum to change with time (Talon \& Charbonnel, 2005; Alvan et al., 2013). Such a specific rotation profile, if detected, would indicate 


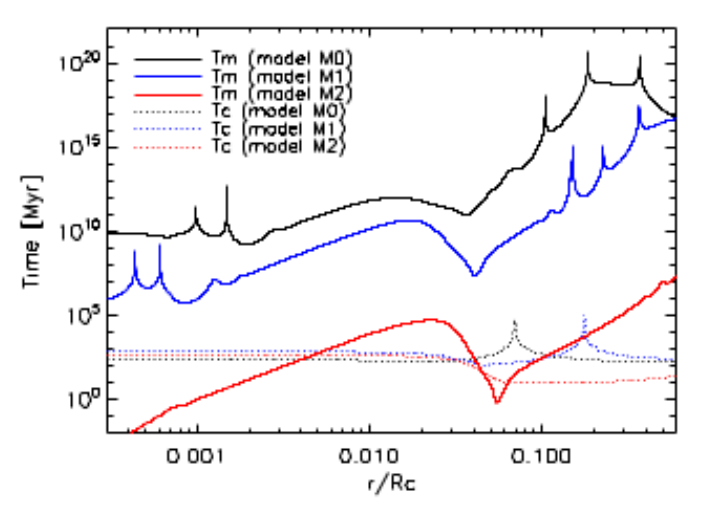

Figure 23: Mixed mode AM transport timescales (solid lines) versus radius normalised to the radius of the base of the convective envelope for one subgiant model, one model at the bottom of the RGB and one RGB model located just below the RG bump. The dotted lines correspond to the timescale associated with the evolution of the star (Credit Belkacem et al 2015b).

that IGW are operating efficiently in evolved stars, however the possibility of a seismic detection of a IGW induced rotation gradient will depend on the location of the front within the star when we observe the star. If the front happens to be in the intermediate evanescent region of the star, it will likely not be detected.

In any case, for red giant stars, the IGW damping becomes so large that IGW seem unable on their own to decrease the core rotation of RGB (Pincon et al., 2017). At this evolutionary phase, another AM transport mechanism must operate.

Transport by mixed modes Prograde and retrograde mixed modes are differentially damped in presence of rotation, allowing a net transport of angular momentum (Townsend, 2014 and references therein). Building on that picture, Belkacem et al. (2015a) investigated the AM transport by mixed modes of stars in post main sequence phases. Belkacem et al. (2015b) then estimated the rate of angular momentum transported by mixed modes for $1.3 M_{\odot}$ models in the subgiant and red giant phases. The AM flux is found to increase with evolution from the subgiant to the top of the red giant branch due to a combination of several factors. The efficiency of the AM transport by modes was then assessed by comparing the timescale associated with the transport of angular momentum by mixed modes with the time scale associated with the core contraction of the star. The comparison is shown in Fig.23 which clearly indicates that the AM transport process is inefficient (too slow) for subgiants and giants at the base of the RGB but is able to counterbalance the structural changes for a giant higher up on the RGB. A more quantitative investigation requires evolutionary calculations including the effect of mixed modes together with other identified AM transports which are yet to be done. This will allow to check a posteriori assumptions made in deriving the prescription for AM transport by mixed modes.

Magneto-hydrodynamic instabilities The Tayler magnetic instability is believed to occur in stellar interiors (Spruit, 1999; Goldstein et al, 2018). What is still debated is the existence of the TaylerSpruit dynamo mechanism. In any case, assuming the prescription proposed by Spruit (2002) for the AM diffusivity, the Tayler-Spruit dynamo was shown to be not efficient enough to counterbalance the core acceleration due to its contraction with evolution for the red giant stars (Cantiello et al. 2014). Fig.24 shows the rotation period as a function of the radius for the subgiant and stars on the red giant branch. These observations are compared to the evolution of the rotation period with the radius for 


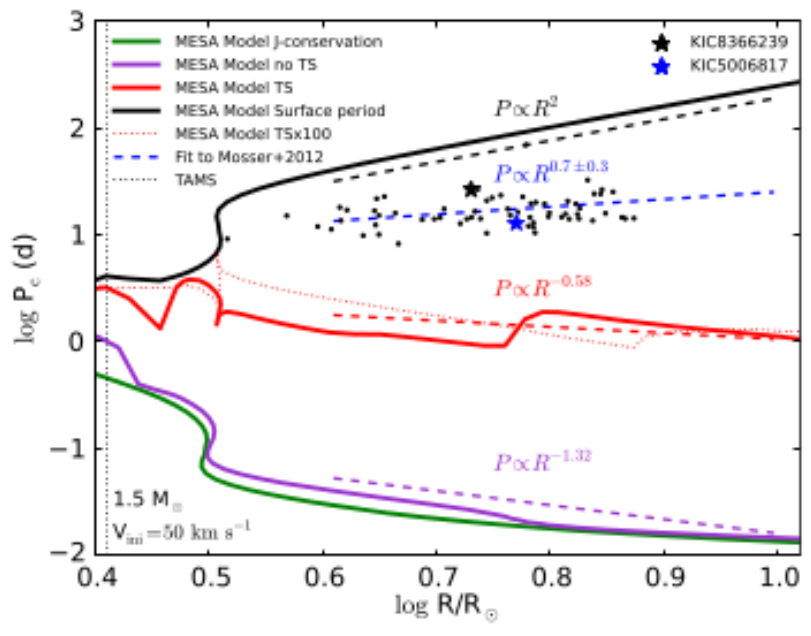

Figure 24: PoMS evolution of the core rotation period as a function of the stellar radius for a $1.5 M_{\odot}$ model assuming different assumptions for AM transport: no AM transport (green), AM transport due rotational instabilities (purple) and due to magnetic torques in radiative regions (red) and a case when the Tayler-Spruit diffusion coefficient has been multiplied by a factor of 100 (red dotted line). Observations (Mosser et al., 2012, Beck et al., 2012) are represented as dots (Credit Cantiello et al., 2014).

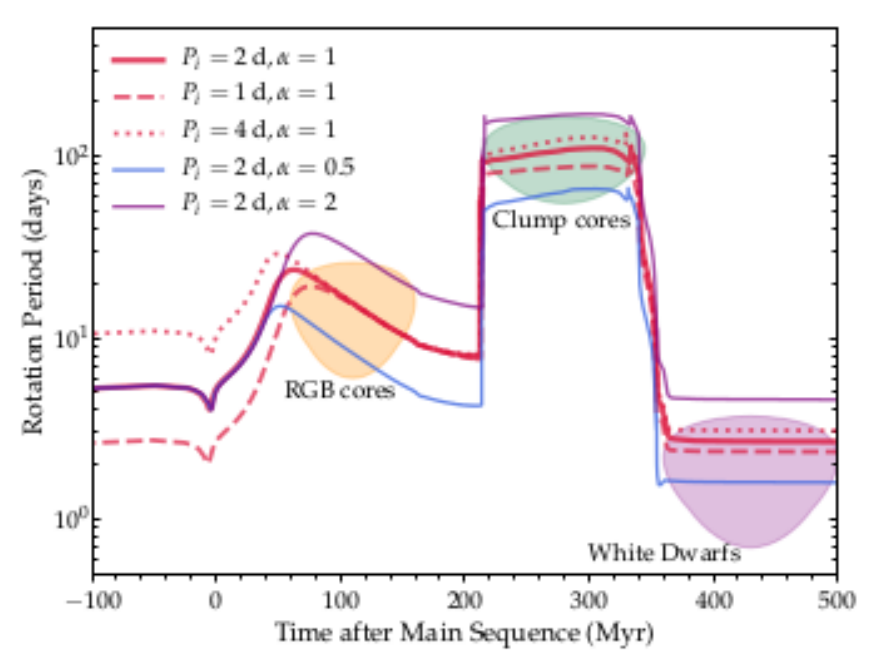

Figure 25: PoMS evolution of the core rotation period as a function of the the time after main sequence for a $1.6 M_{\odot}$ model including the revised prescription for the magnetic diffusivity related to a Taylor instability. The core rotation rates are represented for different values of the parameters. The coloured areas define the observed range for $R G B, R G$ and WD phase (for the later, Hermes et al. 2017) (Credit Fuller et al., 2019).

different types of stellar models built assuming different assumptions about the internal AM transport. Fig.24 shows that they all fail to reproduce the observations.

Recently, Fuller et al. (2019) revisited the saturation mechanism of the Taylor instability. As a result, the magnetic field can grow to larger amplitudes, leading to a larger magnetic torque and therefore to a larger AM diffusivity $\nu_{B} \sim r^{2} \Omega^{3} / N_{\mu}$ where $N_{\mu}$ is the dominant component of the BrüntVaissälä due to a chemical gradient. The authors then showed that this new prescription implemented in an evolutionary code is able to reproduce the magnitude of the low RGB core rotation rates, clump stars and white dwarfs (Fig.25). Prior to these stages, nearly rigid rotation in the radiative zone is maintained during and beyond the end of the MS during the early subgiant phase. However the model predicts a spin down for the late subgiants and a spin up evolution for the red giants when the TS instability can no longer hold the acceleration due to contraction. These results are then at odds with the present observations that-is the core spin up of the subgiants with evolution and the core spin down (Mosser et al 2012) or a constant spin evolution (Gehan et al. 2018) found for the RGB stars. It is not impossible that the conclusions drawn from observations continue to evolve when a larger sample of higher quality data become available as the results from Mosser et al. (2012) then Gehan et al. (2018) showed. This can be particularly true for subgiant stars since the present sample is quite small.

Another magnetic instability, the Magneto-Rotational Instability (MRI) is rather triggered by a radial differential rotation and can lead to AM transport (Rüdiger et al., 2014, 2015). Jouve et al (2015) studied the coupled evolution of a magnetic field and differential rotation in an 3D spherical unstratified radiative shell and found that the MRI developes when the rotational shear is large enough. The rotational shear must overcome the stable stratification so it is not clear yet if the MRI can develope in the stellar rotation/stable stratification regime. Spada et al. (2016) computed the evolution of the rotation profile of a 1.2 $M_{\odot}$ model including AM transport in radiative interior which assumes 

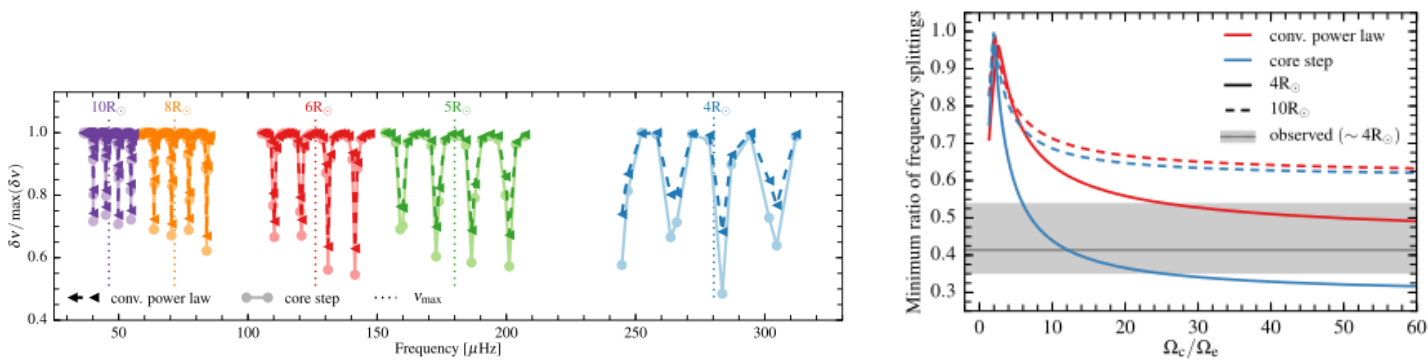

Figure 26: Left: rotational splittings normalized to their maximal values for $1.33 M_{\odot}$ red giant models assuming either a two-step rotation profile with the radial differential rotation just outside of the hydrogen burning shell (lighter solid lines) or a rotation profile varying as a power law with radius in the convection zone and uniformly in the radiative region (darker dashed lines). Cases are labelled with the stellar radius of the model for a proxy of evolution. The ratio $\Omega_{\text {env }} / \Omega_{\text {core }}=7.89$ is taken the same in all cases. The dotted lines show $\nu_{\text {max }}$, indicator ot the frequency at maximum power for each model. Right: Local splitting minima normalized to the maximum splitting value as a function of the rotation ratio $\Omega_{\text {env }} / \Omega_{\text {core }}$ for several rotation profiles: a two zone model (blue line) and a model assuming a uniform rotation in the radiative zone and a power law decrease in the convective envelope (red line). The grey area indicates the range of observed values.(credit Klion \& Quataert, 2017).

an ad-hoc dependence for the AM diffusion coefficient on rotation core-to-surface ratio. The authors found that a dependence of $\left(\Omega_{\text {rad }} / \Omega_{\text {env }}\right)^{3}$ reproduces the observations and is consistent with what could be expected from an AMRI (Azimuthal Magneto-Rotational Instability instability, Rüdiger et al. 2015). Note that this latter dependency- scaling with the differential rotation- differs from the previous TS one which behaves as $\sim r_{\text {core }}^{2} \Omega_{\text {core }}^{2} / N_{\mu}$ near the H-burning shell where the chemical gradient is large.

Conclusion: During the rapid evolution of the PoMS phases, the AM hydrodynamical (rotation related) transport is inefficient to compensate for the strong core rotation increase due to the contraction of the inner layers with evolution. Some missing AM transport must operate to account for the observations. While theoretical developments aim at modelling physically the AM transport candidates, other studies go along another direction and work at - characterizing further the observed rotation profiles namely the location of the internal differential rotation and - characterizing observationally the missing AM transport as a function of stellar parameters and evolutionary phases. These are briefly addressed in the following subsection.

\subsection{Attempts to characterize further the rotation profile : location of the radial differential rotation}

We have seen that the rotation core-to-envelope ratio of PoMs stars is smaller than expected but remains large. These stars then maintain some degree of radial differential rotation. The candidate mechanisms for AM transport in postMS stars are able to generate more or less sharp radial rotation gradients which can be located at different places in the star. When hydrodynamical processes and fluid instabilities dominate, the convective envelope is assumed to rotate uniformly and a sharp rotation gradient developes near the hydrogen burning shell in the radiative region as shown in Fig.20. On the other hand, considering magneto-hydrodynamic instabilities and core-envelope coupling to be responsible for shaping the rotation of red giants, Kissin \& Thompson (2015) studied the case when the radial differential rotation resides in the convection zone and the radiative interior is rotating uni- 
formly. When the impact of the Tayler instability dominates, a sharp rotation gradient developes near the hydrogen burning shell in the radiative region as for the pure hydrodynamical case but with a much smaller rotation gradient (Fuller et al. 2019). If IGW dominates, the rotation gradient moves outward on secular time scales and can be catched up anywhere in the star depending at which phase we observe the star (Alvan et al. 2013). An important hint for finding out what is the dominant AM transport mechanism can then come from the localization of the radial differential rotation. Seismic diagnostics able to locate the rotation gradient are therefore of tremendous importance. Several methods can be used which indicate - first that the seismic information is localized in the central regions and at the very surface; in between not enough information with the detected $\ell=1$ modes is available to resolve the rotation gradient in the intermediate regions - second the signature of the location of differential rotation is easier to detect for less evolved red giants. Attention then concentrated on subgiants and early red giants.

The simplest method assumes a step rotation profile in a two-zone model where an average rotation is assumed in the inner part, $\Omega_{\text {core }}$, and one average value, $\Omega_{e n v}$, is assumed in the envelope. In a forward approach, the impact of the position of the rotation gradient was then investigated on the rotational splittings (Deheuvels 2014, 2015, Triana et al 2017). Klion \& Quataert (2017) suggested a simple seismic diagnostic to locate the radial differential rotation. They showed that at least for the smallest red giants the quantity $\delta \nu_{\min } / \delta \nu_{\max }$ evaluated near $\nu_{\max }$ is sensitive to whether the radial differential rotation is located in the radiative region above the $\mathrm{H}$ burning shell or concentrated within the convective envelope. The splittings of the p-dominated modes $\delta \nu_{\min }$ normalized to the maximum splitting $\delta \nu_{\max }$ (i.e. of g-dominated modes) indeed are smallest for the radial differential rotation located in the radiative region. Fig. 26 (left) taken from Klion \& Quataert (2017) indicates that the diagnostic is more efficient for stars with about 4- 5 stellar radius. Fig. 26 (right) plots $\delta \nu_{\min } / \delta \nu_{\max }$ as a function of $\Omega_{\text {core }} / \Omega_{e n v}$ and illustrates the fact that for most early giant stars the assumption of the radial differential rotation located in the radiative region is favoured.

Using Eq.32 with various rotation profiles, it is possible to explain the decrease of $\delta \nu_{\min } / \delta \nu_{\max }$ with the rotation core-to-envelope (Fig. 26 (right)) and the signature of a different location of the differential rotation. As in Klion et al. (2017), I consider two rotation profiles.

- A first rotation profile assumes that the rotation gradient is located within the g-cavity at a radius $r_{i}\left(r_{a}<r_{i}<r_{b}\right)$ and is uniform below and above that-is

$$
\Omega(r)=\left\{\begin{array}{cc}
\Omega_{c} & \text { for } r \leq r_{i}<r_{b} \\
\Omega_{e} & \text { for } r_{i} \leq r
\end{array}\right.
$$

The core rotation averaged over the $\mathrm{g}$-cavity and the envelope rotation averaged over the $\mathrm{p}$ cavity can be respectively approximated as (Goupil et al, 2013)

$$
\begin{aligned}
\Omega_{\text {core }} & =\frac{1}{\int_{r_{a}}^{r_{b}} \frac{N}{r} d r} \int_{r_{a}}^{r_{b}} \Omega \frac{N}{r} d r \\
\Omega_{\text {env }} & =\frac{1}{\int_{r_{c}}^{r_{d}} \frac{d r}{c_{s}}} \int_{r_{c}}^{r_{d}} \Omega \frac{d r}{c_{s}}
\end{aligned}
$$

For the present purpose, it is enough to assume that the Brünt -Väissälä frequency in the gcavity is $N \sim N_{\text {core }}=$ const and that in the p-cavity the inverse of the sound speed dominates close to the surface $1 / c_{s} \sim 1 / c_{R}=$ const. One then obtains

$$
\begin{aligned}
\Omega_{\text {core }} & \sim \Omega_{c} \frac{\log \left(r_{i} / r_{a}\right)}{\log \left(r_{b} / r_{a}\right)} \\
\Omega_{\text {env }} & \sim \Omega_{e}
\end{aligned}
$$


This gives for the rotation ratio

$$
\mathcal{R}=\frac{\Omega_{\text {env }}}{\Omega_{\text {core }}}=\frac{\Omega_{e}}{\Omega_{c}} \frac{\log \left(r_{b} / r_{a}\right)}{\log \left(r_{i} / r_{a}\right)}
$$

and for the splitting ratio (Eq.32):

$$
\frac{\delta \nu_{\text {min }}}{\delta \nu_{\max }} \sim \frac{1}{1+1 /(q \mathcal{N})}\left(1+\frac{2}{q \mathcal{N}} \frac{\Omega_{\text {env }}}{\Omega_{\text {core }}}\right)=\frac{1}{1+1 /(q \mathcal{N})}\left(1+\frac{2}{q \mathcal{N}} \frac{\log \left(r_{b} / r_{a}\right)}{\log \left(r_{i} / r_{a}\right)} \frac{\Omega_{e}}{\Omega_{c}}\right)
$$

The splitting ratio linearly increases with $\Omega_{e} / \Omega_{c}$ or when considering the $\Omega_{c} / \Omega_{e}$ ratio as in Klion et al. (2017) (Fig.26) decreases with $\Omega_{c} / \Omega_{e}$ as $1 /\left(\Omega_{c} / \Omega_{e}\right)$ with a rate

$$
\text { rate }_{1}=\frac{2}{q \mathcal{N}} \frac{\log \left(r_{b} / r_{a}\right)}{\log \left(r_{i} / r_{a}\right)} \sim \frac{2}{q \mathcal{N}}
$$

The last equality is obtained for the $4 R_{\odot}$ model of Klion et al. (2017) for which one has for the radii normalized to the stellar radii $r_{a}<<10^{-4} ; r_{i} \sim 0.01 ; r_{b} \sim 0.1 ;=r_{B Z C} \sim 0.2 ; r_{c} \sim$ $0.3, r_{d} \sim 1$. Since $r_{a}<<r_{i}<<r_{b}$, so that $\ln \left(r_{b} / r_{a}\right) / \ln \left(r_{i} / r_{a}\right)>1$ goes to 1 when $r_{a}$ goes to zero.

- For a rotation gradient in the convective region, a simplified rotation profile can be assumed of the form

$$
\Omega(r)=\left\{\begin{array}{cc}
\Omega_{c} & \text { for } r \leq r_{B Z C} \\
\frac{\Omega_{e}}{r^{\alpha}} & \text { for } r_{B Z C}<r
\end{array}\right.
$$

For sake of simplicity, $\alpha=1$ hereafter, then

$$
\begin{aligned}
\Omega_{\text {core }} & \sim \Omega_{c} \\
\Omega_{\text {env }} & \sim \Omega_{e} \frac{\ln \left(1 / r_{B Z C}\right)}{1-r_{B Z C}}
\end{aligned}
$$

This gives for the rotation ratio

$$
\mathcal{R}=\frac{\Omega_{\text {env }}}{\Omega_{\text {core }}}=\frac{\Omega_{e}}{\Omega_{c}} \frac{\ln \left(1 / r_{B Z C}\right)}{1-r_{B Z C}}
$$

and for the splitting ratio (Eq.32):

$$
\frac{\delta \nu_{\min }}{\delta \nu_{\max }} \sim \frac{1}{1+1 /(q \mathcal{N})}\left(1+\frac{2}{q \mathcal{N}} \frac{\ln \left(1 / r_{B Z C}\right)}{1-r_{B Z C}} \frac{\Omega_{e}}{\Omega_{c}}\right)
$$

The splitting ratio again linearly increases with $\Omega_{e} / \Omega_{c}$ or when considering the $\Omega_{c} / \Omega_{e}$ ratio as in Klion et al. (2017) decreases with $\Omega_{c} / \Omega_{e}$ as $1 /\left(\Omega_{c} / \Omega_{e}\right)$. with a rate

$$
\text { rate }_{2}=\frac{2}{q \mathcal{N}} \frac{\ln \left(1 / r_{B Z C}\right)}{1-r_{B Z C}} \sim \frac{2}{q \mathcal{N}} 2
$$

As above, the last equality is obtained for the $4 R_{\odot}$ model of Klion et al. (2017).

The ratio rate $_{2} /$ rate $_{1} \sim 2$ indicates that the splitting ratio $\delta \nu_{\min } / \delta \nu_{\max }$ decreases from 1 with $\Omega_{c} / \Omega_{e}$ more rapidly for the core step rotation profile than for a convective power law dependence of the rotation. We recover the results found numerically by Klion et al. (2017). 

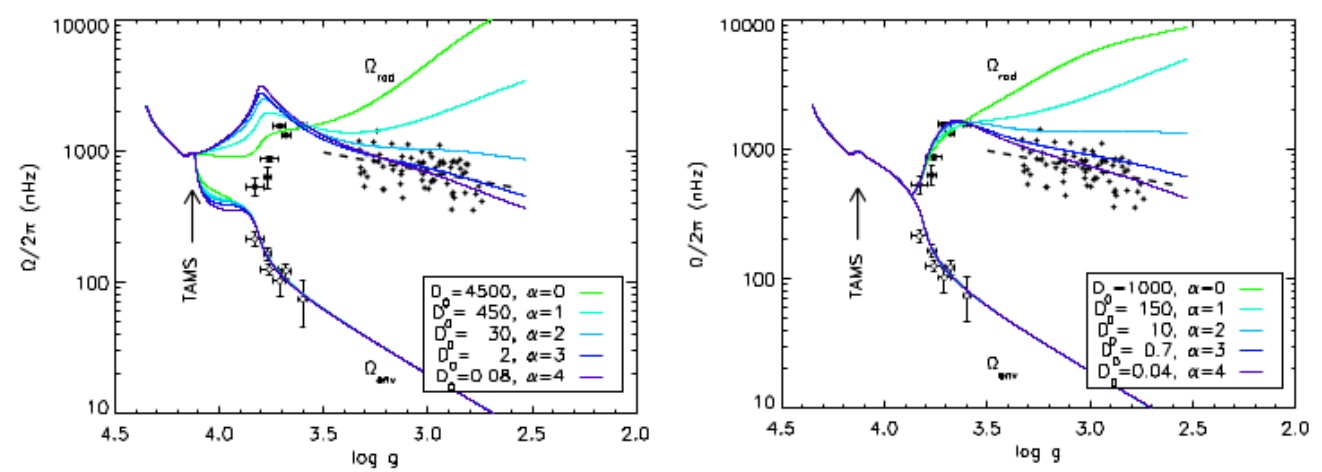

Figure 27: right: evolution of the core rotation rate assuming a AM diffusive transport coefficient scaling as $\left(\Omega_{\text {rad }} / \Omega_{\text {core }}\right)^{\alpha}$ and a enforced uniform rotation during the main sequence (left) and maintained on the subgiant phase until slightly before the first dredge starts (credit Spada et al. (2016)).

Note that a differential rotation causes $\delta \nu_{\max } \approx \Omega_{\text {core }} /(2 \pi)$ to take also different values for a same $\Omega_{e} / \Omega_{c}$ ratio. This means that a least in theory a plot $\delta \nu_{\min } / \delta \nu_{\max }$ as a function of $\delta \nu_{\max }$ ought to discriminate between two different differential rotations. This will be tested with a large sample of stars with similar masses and radii to try to reach some general conclusions about the location of the rotation gradient. An independent measure of the surface rotation such as that obtained with a rotational modulation of the light curve would of course be invaluable in that respect.

More sophisticated methods such as inversion of the rotational splittings can also be used (Deheuvels et al. 2012, 2014, 2015; Di Mauro et al., 2016; Triana et al., 2017). For instance, inversions performed by Deheuvels et al. (2014) showed that a sharp variation of the rotation profile near the $\mathrm{H}$ burning shell is favored over a smooth profile at least for two Kepler early red giants. Triana et al. (2017) used the rotational splittings of 13 out of the 19 red giants analyzed by Corsaro et al. (2015) to derive their average rotation rates. The stars are in a similar evolutionary stage (with a radius $R \sim 5 R_{\odot}$ ) and their core - to - surface rotation ratios are found in the range 5-10. For one star, Triana et al. (2017) derived the rotation profile using the various aforementionned methods and found that they essentially give the same results and concluded that -with observations currently at at hand- no precise information can be obtained in the radiative region above the H-burning shell. The authors also conclude that the Klion \& Quataert (2017) diagnostic is inefficient to distinguish between a radial differential rotation either in the radiative or in the convective region for the considered stars.

\subsection{Attempts to characterize the underlying AM transport process : efficiency of the missing mechanism(s)}

A series of work was carried out with the aim of characterizing the efficiency of the unidentified AM transport mechanism operating in PoMS stellar interiors. This was achieved by including an additional constant viscosity $\nu_{a d d}$ to the AM transport equation i.e. $\nu_{v}=\nu_{\text {shear }}+\nu_{a d d}$ in Eq.36 and ajusting the values $\nu_{a d d}$ in order to reproduce the observations. Such studies were performed for a $1.5 M_{\odot}$ red giant KIC 8366239 (Eggenberger et al. 2012), a $0.84 M_{\odot}$ red giant KIC 7341231 (Eggenberger et al., 2015) as well as for the observed subgiants (Eggenberger et al., 2018) . It was found that when constraints on both the core and surface rotation rates are available- which is particularly true for subgiants- a precise value can be obtained for $\nu_{a d d}$. Indeed while the knowledge of the core rotation rate imposes a sufficiently high value of $\nu_{\text {add }}$ so that it dominates the AM transport, a constraint on the surface rotation rate limits the maximal value of this added viscosity. These precise determinations led to the conclusions that the efficiency of the missing AM transport as represented by $\nu_{a d d}$ must 
increase with the mass in order to reproduce the observations. For instance, $\nu_{\text {add }}$ for KIC 7341231 is at least three times lower than the one obtained for KIC 8366239 independently of their different evolution stage. Using the observations of a large sample of red giant stars (Mosser et al., 2012) and a sample of subgiant stars (Deheuvels, 2015), it was also found that the behavior of $\nu_{a d d}$ with evolution differs during the subgiant and the red giant branch: the efficiency decreases on the subgiant branch and increases during the red giant ascending branch (Cantiello et al., 2014; Eggenberger et al., 2015; Spada et al., 2016, Eggenberger et al., 2018). This conclusion closely follows what is expected from the observations when the core rotation of the subgiants increases whereas the core of the ascending red giant decreases despite the core contraction (Deheuvels 2015). Another important information brought by these studies is that the value of $\nu_{a d d}$ is not sensitive to the past rotation history of the star, except at the very beginning of the subgiant phase and can therefore probe the AM transport taking place during the post MS phase.

In another approach, Spada et al. (2016) computed the evolution of the rotation profile of a 1.25 stellar model up to the RGB phase including internal AM transport as a pure diffusive transport $\left(U_{2}=0\right)$ in Eq.36. Surface AM losses were taken into account according to the Kawaler (1988)'s prescription. On one hand, the Kawaler's constant $K_{w}$ is adjusted so that the models can reproduce the observed envelope rotation of the subgiants and red giants. On the other hand, the authors assumed a turbulent diffusion for the internal AM transport of the form

$$
D=D_{0}\left(\frac{\Omega_{\text {rad }}}{\Omega_{\text {core }}}\right)^{\alpha}
$$

where $D=\nu_{v}$ in our notation (Eq.36). The exponant $\alpha$ was determined so that the evolutionary stellar models can reproduce the observed core rotations of red giants and their evolution. Motivated by the fact that observations of main sequence stars indicate a rigid rotation for several stars besides the Sun (Nielsen et al., 2014; Benomar et al., 2015), Spada et al (2016) studied first the case when a rigid rotation is enforced on the main sequence until the TAMS. The best match for the red giant core evolution is obtained for a value $\alpha=3$, which as already mentioned (Sect.4.2) may have some physical justification. However, the evolution of the core rotation on the subgiant phase is not reproduced correctly: the core accelerates too rapidly (Fig.27(left)). In a second computation, the rigid rotation is imposed until a point where the core rotation can be well reproduced for all subgiants. Spada et al. (2016) identified this time as roughly the time when the hydrogen burning shell has been fully extended before the convective envelope start to recede. Then a single set of parameter values for $(\alpha, D)$ is able to reproduce the evolution of the subgiant and red giant branches (Fig.27(right)). The data for the red giants were taken from Mosser et al. (2012), some small ajustement of the parameter values might be necessary in order to agree with the smaller spin down found by Gehan et al. (2018).

Eggenberger et al. (2018) find a similar behavior when computing the AM transport according to Eq. 34 including meridional circulation and shear induced turbulence and including an additional viscosity $\nu_{a d d}$ as mentionned previously. The values of the additional viscosity and the rotation period at the ZAMS are ajusted for each subgiant so that the core and surface rotation of the models reproduce simultaneously the observed ones. The additional viscosity is added first from the very of beginning of the evolution of the model (Fig.28(left)). Following the suggestion by Spada et al. (2016), Eggenberger et al. (2018) also evolved the models for each subgiant enforcing a rigid rotation on the MS and beyond the TAMS until a point where the rigid rotation is turned off and the contracting core is free to accelerate up to the observed value. In that case, no additional AM transport than meridional circulation and shear turbulence is necessary on the subgiant branch (Fig.28(left)). The issue here is that according to the authors, the time at which the rigid rotation needs to be turned off differs for each subgiant and does seem to appear at a specific phase of the subgiant evolution. 

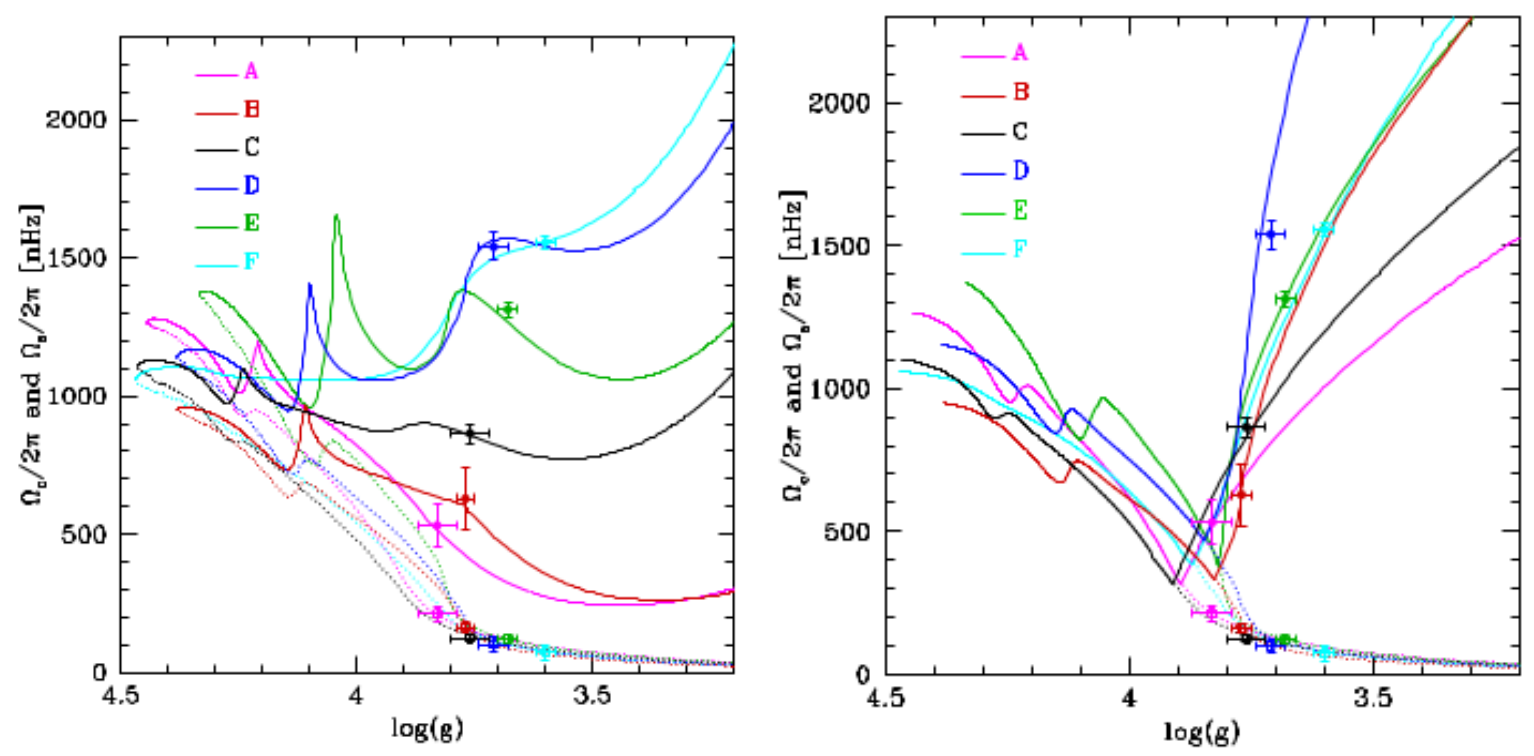

Figure 28: left: Core (solid lines) and surface (dotted lines) rotation rates as a function of gravity for rotating models of the six subgiants determined by Deheuvels et al. 2014. Dots and open circles indicate the seismic values of core and surface rotation rates determined for the six subgiants. Right same as left but the models are computed by assuming solid-body rotation until a given point during the post-main sequence. (Credit Eggenberger et al., 2019)

\section{Conclusions}

Significant advances in Galactic archeology were brought up by the ability of the seismology of red giants to constrain precisely their masses and ages. At the same time, the seismic probing of the internal properties of these stars revealed severe flaws in our understanding and modelling of the structure and the internal AM evolution of the red giants. This motivated a vivid brainstorming activity in the community leading to several valuable advances in the field. In this review, I chose to address two important issues: mixing beyond the convective core of red clump stars and angular momentum transport in low mass post main sequence stars. In a nutshell, what can be concluded?

On the structural side, the observed period spacings of red clump stars confirm that these stars have quite larger, more massive convective cores than predicted by the classical Schwarzschild criterion as implemented in many stellar evolution. The properties of the extra-mixing region above the formal convective core can in principal be determined as well. The contribution of the Liège school led by Arlette Noels has been determinant in the significant advances of the past decade for that challenging field. However the way forward from the seismic observations to a proper modelling of the central regions of CHeb stars is not easy and the final word is not said yet. To proceed beyond a mere ajustement of a free parameter, one must await significant advances from numerical simulations and theoretical developments. On the observational side, the previous studies have shown the necessity of a larger sample of stars to cover the range of mass, evolution and metallicity.

The general picture of AM transport is nowadays certainly more satisfying since it is now possible to reconciliate the core rotation rates of the red giant stars and the rotation rates of their descendants, the white dwarfs. Several processes have been identified as likely candidates for the AM evolution of PoMS star. However a tremendous amount of work is still awaiting ahead of us to confirm these ideas, this must be a combination of theoretical developments, numerical simulations and data analyses. Indeed a full modelling of the total AM flux along the evolution must now include all these processes which might operate and dominate at different phases of the star evolution $\mathcal{F}_{\text {tot }}=\mathcal{F}_{\text {circ }}+\mathcal{F}_{\text {shear }}+$ 
$\mathcal{F}_{\text {waves }}+\mathcal{F}_{\text {modes }}+\mathcal{F}_{B}$. Prescriptions for the transport coefficients for each individual process are nowadays included and studied individually on more physical grounds but certainly deserve further improvements. The interations between these processes ought now to be taken into account and quantified by their consistent implementation in 1D stellar models. Morover, whereas most of the identified AM transport processes might not have a significant direct impact on the structure and on the frequencies of the axisymmetric $m=0$ modes, the cumulative effect of each dominant process during the evolution of the PoMS might result in some chemical, hence structural, modifications which remain to be investigated and their seismic signatures determined. In that sense, only a few individual stars have been studied so far. On the side of seismic theoretical developements, promising prospects are the study of glitches (Cunha et al., 2015), of the information that $\ell=2$ modes can carry out (Deheuvel et al., 2017), the case of evolved fast rotators (Ouazzani et al., 2013) and the series of investigation on the coupling factor which characterizes the evanescent region between the $g$ and $p$ resonant cavities (Pincon et al., 2019 and reference therein).

While the data from Kepler/K2 have certainly still much more to tell, the currently running NASA mission, TESS (Schofield et al., 2019) and the ESA project PLATO (launch in 2026, Rauer et al., 2014) will offer unvaluable opportunities to address further the above issues.

\section{Acknowledgements}

It was a pleasure to attend the Liege conference for the 75th birthday of Arlette Noels. The present contribution is then dedicated to my dear friend and highly esteemed colleague Arlette. I truely thank Arlette for our lively scientific discussions, her deep understanding of the physics and evolution of stellar interiors and her pedagogical talent to transmit her knowledge and most importantly for our good time and laughs around a diner table. I hope she will forgive me for the involuntary imperfections of the present paper. I also gratefully thank Marc -Antoine Dupret for his careful reading of the manuscript.

\section{References}

Aizenman, M., Smeyers, P., Weigert, A., 1977, AA 58, 41

Alvan, L., Mathis, S., Decressin, T., 2013b, AA 553, A8

Amard, L., Palacios, A., Charbonnel, C. and 2 co-authors, 2016, AA 587, 105

André, Q., Mathis, S., Amard, L., 2018, sf2a. conf 145

Arnett, W. D., Meakin, C., Hirschi, R. et al. 2018, arXiv181004653

Arnett, W. D., Meakin, C., Viallet, M. et al., 2015, ApJ., 809, 30

Baglin, A. and the CoRoT team, 2016, The CoRoT Legacy Book: The adventure of the ultra high precision photometry from space, by the CoRot Team, ISBN: 978-2-7598-1876-1, EDP Sciences, p.1

Baglin, A., Auvergne , M. , Barge, P. et al., 2006, ESASP 1306, 33

Beck, P. G., Montalban, J., Kallinger, T. et al., 2012, Natur, 481, 55

Bedding, T. R., Mosser, B., Huber, D. et al, 2011, Natur, 471, 608

Belkacem, K., Marques, J.P., Goupil, M.J., and 6 co-authors, 2015a, AA 579, A30

Belkacem, K., Marques, J. P., Goupil, M.J., and 6 co-authors, 2015b, AA 579, A31

Benomar, O., 2013 in Progress in Physics of the Sun and Stars: A New Era in Helio- and Asteroseismology ASP Conference Series 479, 161 H. Shibahashi and A. E. Lynas-Gray, eds.

Benomar, O., Belkacem, K., Bedding T. R. et al., 2014, 781, L29

Benomar, O., Takata, M.,, Shibahashi et al., 2015, MNRAS 452, 2654

Borucki , William J., 2018, in Handbook of Exoplanets, ISBN 978-3-319-55332-0. Springer International Publishing AG, part of Springer Nature

Borucki, W. J., Koch, D., Basri, G. et al., 2010, Sci. 327, 977

Bossini, D., Miglio, A., Salaris, M. et al., 2017, MNRAS, 469, 4718

Bossini, D., Miglio, A., Salaris, M. et al., 2015, MNRAS 453, 2290

Brun,, A. S., Palacios, A., 2009, ApJ 702, 1078 
Buysschaert, B., Beck, P. G., Corsaro, E. et al.,2016, A\&A 588, 82

Cantiello , M., Mankovich, C., Bildsten , L. et al., 2014, ApJ 788, 93

Ceillier, T., Eggenberger, P., García, R. A. , Mathis, S., 2013 AA 555, 54

Ceillier, T., Tayar, J., Mathur, S. et al., 2017, AA 605, 111

Chaplin, W. J., Miglio, A., 2013, ARA\&A 51, 353

Charpinet, S., Giammichele, N., Zong, W. et al., 2018, OAst 27, 112

Christensen-Dalsgaard, 2007, Lecture notes on Stellar Oscillations

Corsaro, E., De Ridder, J., García, R. A.,2015, AA 579, 83

Constantino, T., Campbell, S. W., Christensen-Dalsgaard, J. et al., 2015, MNRAS 452, 123

Constantino, T., Campbell, S. W., Lattanzio, J. C.,2017, MNRAS 472, 4900

Cox, J. P., 1980, 'Theory of stellar pulsation',

Cunha, M. S., Stello, D., Avelino, P. P. et al., 2015, ApJ 805, 127

Deheuvels, S. Garca, R. A. Chaplin, W. J., 2012, ApJ 756, 19

Deheuvels, S. Dogan, G. Goupil, M. J., 2014, AA 564, A27

Deheuvels, S., Ballot, J., Beck, P. G., 2015, AA 580, A96

Deheuvels, S., Ouazzani, R. M., Basu, S.,2017, AA 605, 75

Di Mauro, M. P., Ventura, R., Corsaro, E. et al., 2018, ApJ 862, 9

Di Mauro, M. P., Ventura, R., Cardini, D. et al., 2016, ApJ 817, 65

Dupret M.-A., Belkacem K., Samadi R. et al., 2009, AA 506, 57

Dziembowski, W. A., 1971, AcA 21, 289

Dziembowski, W. A.; Gough, D. O.; Houdek, G. et al., 2001, MNRAS 328, 601

Eggenberger, P., Deheuvels, S., Miglio, A. et al., 2019, A\&A 621, 66,

Eggenberger, P., Lagarde, N., Miglio, A. et al., 2017, A\&A 599, 18

Eggenberger, P., 2015, EAS 73, 26

Eggenberger, P., Montalbán, J., Miglio, A., 2012, A\&A 544, 4

Endal, A. S. \& Sofia, S., 1978, ApJ 220, 279

Endal, A. S. \& Sofia, S., 1981, ApJ 243, 625

Fuller, J., Lecoanet, D., Cantiello, M. et al., 2014, ApJ 796, 17

Fuller, J., Piro, A. L., Jermyn, A. S., 2019 MNRAS 485, 3661

Gabriel, M., Noels, A., Montalbán, J. et al., 2014, A\&A 569, 63

Gehan, C., Mosser, B., Michel, E. et al., 2018, AA 616, 24

Goldstein, J., Townsend, R. H. D., Zweibel, E. G., 2018, arXiv180808958

Gough, D. O.,1993, in 'Astrophysical Fluid Dynamics - Les Houches 1987', elsevier science publisher, p. 399

Goupil, M. J., Mosser, B., Marques, J. P. et al., 2013, AA 549, 75

Kawaler, S. D., 1988, ApJ 333, 236

Hekker, S., Christensen-Dalsgaard, J.,2017 ,AAR 25, 1

Hekker, S., Elsworth, Y., Angelou, G. C. et al., 2018, AA 610, 80

Hermes, J. J., Gänsicke, B. T., Kawaler, S. D. et al., 2017, ApJS 232, 23

Jiang, C. \& Christensen-Dalsgaard, J., 2014, MNRAS 444, 3622

Jouve, L., Gastine, T., Lignières, F., 2015, AA 575, 106

Kissin Y., Thompson C., 2015, ApJ, 808, 35

Klion, H. \& Quataert, E., 2017, MNRAS 464, 16

Lecoanet, D. \& Quataert, E., 2013, MNRAS 4302363

Loi, S. T. \& Papaloizou, J. C. B., 2018, MNRAS 4775338

Maeder, A., 2009, 'Physics, Formation and Evolution of Rotating Stars', Springer

Maeder, A. \& Meynet, G., 2004, AA 422, 225

Maeder, A. \& Meynet, G., 2005, AA 440, 1041

Maeder, A. \& Meynet, G., 2014, ApJ 793, 123

Maeder, A., Meynet, G., Lagarde, N. et al., 2013, AA 553, 1

Maeder, A. \& Zahn, J.P., 1998, AA 334, 1000

Marques, J. P., Goupil, M. J., Lebreton, Y. et al., 2013, AA 549, 74

Mathis, S., Zahn, J.-P., 2004, AA 425, 229

Mathis, S, 2013, Transport Processes in Stellar Interiors, LNP 865, 23

Mathis, S \& Alfvan, 2013, Transport Processes in Stellar Interiors, EAS 63, 269

Mathis, S., Prat, V., Amard, L. et al., 2018, A\&A 620, 22

Meynet, G., Ekstrom, S., Maeder, A. et al., 2013, LNP 865, 3

Montalbán, J., Miglio, A., Noels, A. et al., 2013, ApJ 766, 118 
Montalbán, J., Noels, A., 2014, in 'Asteroseismology of red giant stars: The potential of dipole modes ', EPJWC 43

Mosser, B., Barban, C., Montalbàn, J., et al. 2011, AA, 532, A86

Mosser, B., Goupil, M. J., Belkacem, K., 2012a, AA 540, A143

Mosser, B., Goupil, M. J. Belkacem, K., 2012b, AA 548, A10

Mosser, B.,, O. Benomar, K. Belkacem, 2014, AA 572

Mosser, B., Vrard, M., Belkacem, K. et al., 2015, AA 584, 50

Mosser, B., Miglio, A. and CoRot Team, 2016, in The CoRoT Legacy Book: The adventure of the ultra high precision photometry from space, by the CoRot Team . ISBN: 978-2-7598-1876-1. EDP Sciences , p.197

Mosser, B., Pinçon, C., Belkacem, K. et al., 2017, AA 607, 2

Mosser, B., Gehan, C., Belkacem, K. et al., 2018, AA 618, 109

Nielsen, M. B., Gizon, L., Schunker, H. et al., 2014, A\&A 568, 12

Noels, A., Montalban, J., Miglio, A. et a., 2010, ApSS 328, 227

Noels, A., 2013, LNP 865, 209

Ouazzani, R. -M., Goupil, M. -J., Dupret, M. -A. et al., 2013, ASPC 479, 335

Palacios, A., 2013, EAS 62, 227

Paxton, B., Schwab, J., Bauer, E. B. et al., 2018, ApJS 234, 34

Paxton, B., Smolec, R., Schwab, J. et al., 2019, arXiv190301426P,

Pinçon, C., Belkacem, K., Goupil, M. J., 2016, AA 588, 122

Pinçon, C., Belkacem, K., Goupil, M. J. et al., 2017, AA 605, 31

Pinçon, C.,Takata, M., Mosser, B., 2019, arXiv190505691

Pinsonneault, M. H., Kawaler, S. D., Sofia, S. et al., 1989, ApJ 338, 424

Press, W. H., 1981, ApJ 245, 286

Rauer, H., Catala, C., Aerts, C. et al., 2014, ExA 38, 249

Rüdiger, G., Gellert, M.,Spada, F. et al., 2015, AA 573, 80

Rüdiger, G., Schultz, M., Kitchatinov, L. L.,2014, arXiv1404.3562

Salaris, M. \& Cassisi, S., 2017, RSOS 4, 170192

Schatzman, E., 1993, AA 279, 431

Scuflaire, R. ,1974, AA 34, 449,

Schofield, Mathew, Chaplin, W. J., Huber, D. et al., 2019, ApJS 241, 12

Shibahashi, H., 1979, PASJ 31, 87

Spada, F., Gellert, M., Arlt, R. et al., 2016, AA 589, 23

Spruit, H. C., 1999, AA 349, 189

Spruit, H. C.,2002, AA 381, 923

Spruit, H. C.,2015, AA 582, L2

Takata, M., 2016a, Publ. Astron. Soc. Japan 68, 6

Takata, M., 2016b, Publ. Astron. Soc. Japan 68, 91

Talon, S. \& Zahn, J. -P., 1997, AA 317, 749

Talon, S., 2004, IAUS 215, 336

Talon, S., 2008, EAS 32, 81

Talon, S., \& Charbonnel, C. 2005, AA, 440, 981

Talon, S. \& Charbonnel, C., 2008, AA 482, 597

Tayar, J., Ceillier, T., Garca-Hernndez, D.A., 2015, ApJ 807, 82

Townsend, R., 2014, in IAU Symp. 301, eds. J. A. Guzik, W. J. Chaplin, G. Handler, A. Pigulski, 153

Triana S. A., Corsaro E., De Ridder J. et al., 2017, AA, 602, A62

Unno, W., Osaki, Y., Ando, H., Saio, H., Shibahashi, H. , 1989 Nonradial oscillations of stars, second edition , University of Tokyo press.

Vrard, M., Mosser, B., Samadi, R. et al., 2016, AA 588, 87

Zahn, J.P., 1991, AA, 252, 179

Zahn, J. P., 1992, AA 265115

Zahn, J.P., Talon, S., Matias, J., 1997, AA 322, 320

Zahn, J.P., 2013, EAS 63, 245 